Publ. RIMS, Kyoto Univ.

15 (1979), 679-718

\title{
Completeness of Modified Wave Operators for Long-Range Potentials
}

$\mathrm{Byy}_{\mathrm{y}}$

Teruo IKEBE and Hiroshi IsOzAKI*

\section{§. Introduction}

Spectral and scattering theory for the Schrödinger operator $H=-\Delta$ $+V$ with $V$ short-range $\left(V(x)=O\left(|x|^{-1-c}\right), \varepsilon>0\right)$ has been developed fairly saisfactorily that one can talk about the existence and completeness of the (ordinary) wave operators

$$
W_{ \pm}=\underset{t \rightarrow \pm \infty}{s-\lim } e^{i t H H} e^{-i t I I_{0}},
$$

where $H_{0}$ is the unperturbed Schrödinger operator $-\Delta$. As compared to this, rather few have been known about long-range scattering. The purpose of the present paper is to give a proof of the completeness of the modified wave operators intertwining $H_{0}$ and $H=-\Delta+V$, where in this case the potential $T$ is long-range, i.e. $V$ is assumed to satisfy the following:

(A) $V(x)$ is a real-valued $C^{\infty}$-function over $\mathbb{R}^{n}$ (=Euclidean $n$-space such that for some $\delta_{0}>0$

$$
D^{a} V(x)=O\left(|x|^{-|\alpha|-\hat{\delta}_{0}}\right) \quad(|x| \rightarrow \infty)
$$

for any multi-index $\alpha=\left(\alpha_{1}, \cdots, \alpha_{n}\right) . \quad\left(C^{\infty}=\right.$ infinitely differentiable; $\left.|\alpha|=\alpha_{1}^{\prime}+\cdots+\alpha_{n} ; D^{a}=\left(\partial / \partial x_{1}\right)^{\alpha_{1}} \cdots\left(\partial / \partial x_{n}\right)^{\alpha_{n}}\right)$.

The formal differential operators $H_{0}$ and $H$ have unique self-adjoint realizations in the Hilbert space $\mathcal{H}=L_{2}\left(\mathbb{R}^{n}\right)$, square integrable functions on $\mathbb{R}^{n}$, which we shall denote again by $H_{0}$ and $H$.

In order to define the modified wave operators we consider appro-

Communicated by S. Matsuura, April 19, 1977.

* Department of Mathematics, Kyoto University. 
priate (approximate) solutions $X_{ \pm}(\xi, t)$ of the (Hamilton-Jacobi) equation

$$
\frac{\partial}{\partial t} X(\xi, t)=V\left(2 \xi t+\nabla_{\xi} X(\xi, t)\right) .^{1)}
$$

By "appropriate" we mean that they should be "well-behaved" asymptotically. (The precise conditions on $X_{ \pm}(\xi, t)$ are specified in Lemma 2.2). We call $X_{ \pm}(\hat{\kappa}, t)$ time-dependent modifiers associated with $H$. With $X_{ \pm}(\xi, t)$ we define the modified wave operators

$$
W_{ \pm}=W_{ \pm}(V)=\underset{t \rightarrow \pm \infty}{\mathrm{s}-\lim _{t \rightarrow \infty}} e^{i t H} e^{-i t H_{0}} e^{-i X_{ \pm}(p, t)}
$$

where $s$-lim means strong limit, and $p$ the differential operator $i^{-1} \nabla_{X}$. It is known that (A) guarantees the existence, isometry and intertwining property of $W_{ \pm}$, and the range of $W_{ \pm}, \operatorname{Ran}\left(W_{ \pm}\right)$, being contained in the subspace of absolute continuity relative to $H, \mathcal{H}_{a c}(H)$ (Buslaev-Matveev [3], Alsholm-Kato [2], Alsholm [1], Hörmander [4]). A harder question is whether $\operatorname{Ran}\left(W_{ \pm}\right)=\mathcal{H}_{a c}(H)$, in which case $W_{ \pm}$are said to be complete. Our goal is to show the following

Theorem 1. 1. Under Assumption (A) there exist time-dependent modifiers $X_{ \pm}(\xi, t)$, and the modified wave operators $W_{ \pm}$are complete.

The idea of proof lies in showing that $W_{ \pm}$equal the so-called stationary modified wave operators $\Omega_{ \pm}=\Omega_{ \pm}(V)$ for which is known the relation $\operatorname{Ran}\left(\Omega_{ \pm}\right)=\mathcal{H}_{a c}(H)$. This will not be carried out in a direct manner, however. First a sequence of potentials $V_{m}$ with compact support are selected which approximate $V$. It is rather easy to show $W_{ \pm}\left(V_{m}\right)=$ $\Omega_{ \pm}\left(V_{m}\right)$. On the other hand, we can show that $W_{ \pm}\left(V_{m}\right) \rightarrow W_{ \pm}(V)$ and $\Omega_{ \pm}\left(V_{m}\right) \rightarrow \Omega_{ \pm}(V)$ as $m \rightarrow \infty$. Thus the desired conclusion follows by passing to the limit for $m \rightarrow \infty$ in $W_{ \pm}\left(V_{m}\right)=\Omega_{ \pm}\left(V_{m}\right)$.

The construction of $\Omega_{\Perp}$ is based on a spectral representation or eigenfunction expansion: One can find unitary operators $\mathscr{E}_{ \pm}$from $\mathscr{H}$ onto $\widehat{\mathcal{H}}=L_{2}\left(\mathbb{R}_{+}: L_{2}\left(S^{n-1}\right)\right) \quad($ see $\S 5)$ such that $\mathscr{F}_{ \pm} H=M \mathscr{F}_{ \pm}$, where $M$ is the

1) The subscript variable as in $\nabla_{\xi}, \nabla_{x}$ indicates the variable in question for definiteness' sake though not logically necessary. 
multiplicative operator by the independent variable on $\mathbb{R}_{+}$; the stationary wave operators $\Omega_{ \pm}$are defined by $\Omega_{ \pm}=\mathscr{F}_{ \pm}^{*} \mathscr{F}_{0 \pm}$ ( $\mathscr{F}_{0 \pm}$ is the $\mathscr{F}_{ \pm}$for $V$ $=0)$. Although a spectral representation theory has been developed previously by Ikebe [5] and Saitō [11], we shall propose a new approach to spectral representation for $H$ utilizing Isozaki's results [7].

In the sequel we shall restrict ourselves to discussing $W_{+}$, dropping the subscript + , for $W_{-}$can be dealt with in quite a similar way.

Assumption (A) we have imposed on $V$ is not absolutely necessary. For simplicity, however, we have avoided scrupulous examination of the regularity to be required of $V$. The existence of the modified wave operators has actually been proved under weaker conditions on $V$ by the authors referred to above.

Recently, Kitada [9], [10] has proved Theorem 1.1 by a different method. In addition to eigenfunction expansion results his method is based on his definition of stationary modified wave operators, while ours leans over an analysis of time-dependent modified wave operators.

The contents of the present paper are as follows. Section 2 discusses time-dependent modifiers $X(\xi, t: V)$ and time-dependent modified wave operators $W(V)$, and the continuous dependence on $V$ of these quantities. In Section 3 we state some known results from Ikebe-Saitō [6] concerning the so-called limiting absorption principle in which the boundary values $R(\lambda \pm i 0: V)$ for $\lambda$ real of the resolvent of $H=H(V)$ are our main theme. The continuous dependence on $V$ of $R(\lambda \pm i 0: V)$ is also studied. In Section 4 we transform the time-dependent modifiers into the stationary ones via a certain inverse function theorem. Using the stationary modifiers we present in Section 5 an eigenfunction expansion theory and a stationary definition of the modified wave operator $\Omega(V)$. In Section 6 $\Omega(V)$ is shown to depend continuously on $V$. In Section 7 is proved the relation $W(V)=\Omega(V)$ for compactly supported potentials $V$. Section 8 completes the proof of Theorem 1.1.

\section{$\S 2 . \quad$ Time-Dependent Mlodified Wave Operators}

In this section we present the results of Buslaev-Matveev [3], Alsholm [1] and Hörmander [4] on the existence of time-dependent modi- 
fied wave operators, and prove their continuous dependence on the potential $V(x)$.

First we introduce a function space $\mathbb{V}$ of potentials.

Defimition 2. $\mathbb{H}_{\text {. Let }} 0<\delta<1$. Let $\mathbb{V}=\mathbb{V}_{\delta}$ be the totality of all real-valued $C^{\infty}\left(\mathbb{R}^{n}\right)$-functions $f(x)$ such that for $N=0,1,2, \cdots$

$$
\|f\|_{\boldsymbol{V}, N} \equiv \max _{|\alpha| \leq N} \sup _{x \in \mathbb{R}^{n}}(1+|x|)^{|\alpha|+\delta}\left|D^{\alpha} f(x)\right|<\infty .
$$

Equipped with norms $\|\cdot\|_{V, N} \mathbb{V}$ is a real Fréchet space. Now we choose a $\delta$ such that $0<\delta<1, \delta<\delta_{0}$ and $\delta$ is irrational (for a technical reason to be made clear in the Appendix). This $\delta$ will be fixed throughout in the present paper. According to our Assumption (A) clearly $V$ $\in \mathbb{V}=\mathbb{V}_{\dot{\delta}}$.

As mentioned in the Introduction, we want to find an (approximate) solution $X(\xi, t: V)$ of the non-linear equation

$$
\frac{\partial}{\partial t} X(\hat{\xi}, t: V)=V\left(2 \xi t+\nabla_{\xi} X(\xi, t: V)\right)
$$

The properties of $X(\xi, t: V)$ needed in the sequel can be summed up in the following lemma whose proof will be given in the Appendix.

Lemma 2. 2. For $V \in \mathbb{V}=\mathbb{V}_{0}$ there exists a function $X(\xi, t: V)$ having the following properties:

(1) $X(\xi, t: V)$ is a real $C^{\infty}$-function of $\xi \neq 0$ and $t>0$.

(2) Let $B$ be a bounded set of $V$ and $K$ a compact set of $\mathbb{R}^{n}-\{0\}$.

Then we have

$$
\begin{gathered}
\left|D_{\xi}^{\alpha} D_{t}^{m} X(\xi, t: V)\right| \leq C(1+t)^{1-m-\grave{o}} \quad(|\alpha| \geq 0, m \geq 0) \\
\left|D_{\xi}^{\alpha}\left[\frac{\partial}{\partial t} X(\xi, t: V)-V\left(2 \xi t+\nabla_{\xi} X(\xi, t: V)\right)\right]\right| \leq C(1+t)^{-2} \\
(|\alpha|=0,1)
\end{gathered}
$$

where the constant $C$ is independent of $\xi \in K, V \in B$ and $t>0$.

(3) Let $V_{n} \rightarrow V$ in $\mathbb{V}$. Then we have for all $\alpha$ and $m D_{\xi}^{\alpha} D_{t}^{m} X\left(\xi, t: N_{n}\right)$ $\rightarrow D_{\xi}^{\alpha} D_{t}^{m} X(\xi, t: V)$ pointwise for $\xi \neq 0$ and $t>0$ as $n \rightarrow \infty$. 
We shall call $\mathrm{X}(\xi, t: V)$ a time-dependent modifier associated with $V$. Before stating the existence results for modified wave operators, we introduce the following notations.

Let $H(V)$ denote the unique self-adjoint realization in $L_{2}\left(\mathbb{R}^{n}\right)$ of $-d+V(x)$, where $V(x) \in V$. In particular $H(0)=H_{0}$. Let $e^{-i X(p, t: v)}$ denote the operator $\left(e^{-i \pi(p, t: v)} u\right)(x)=\mathscr{E}^{-1}\left[e^{-i X(\hat{s}, t: v)} \hat{u}(\xi)\right](x)$, where $\mathscr{E}$ is the ordinary Fourier transform

$$
(\mathscr{F} u)(\xi) \equiv \hat{\imath}(\xi)=(2 \pi)^{-n / 2} \int_{\mathbb{R}^{n}} e^{-i x x^{\frac{1}{\xi}}} u(x) d x .
$$

Theorem 2. 3. Let $V \in \mathbb{V}$. Then the wave operator

$$
W(V) \equiv \underset{l \rightarrow \operatorname{sim}}{ } e^{i t H(V)} e^{-i t I I_{0}-i X(p, t \cdot V)}
$$

exisis, where s-lim means strong limit in $L_{2}\left(\mathbb{R}^{n}\right)$, is a partial isometry with initial set $L_{2}\left(\mathbb{R}^{n}\right)$ and final set contained in $\mathcal{G}_{a c}(H(V))$ and has the interiwining property $H(V) W(V) \supseteq W(V) H_{0}$.

For the proof see e.g. Buslaev-Matveev [3], Alsholm [1] and Hörmander [4].

Now, we shall study the dependence on $V$ of the operator $T(V)$. Set $I V(t: V)=e^{i t H(r)} e^{-i t, \tau_{0}-i . I^{(}(p, t: V)}$.

Lemma 2. 4 . Let $s>0, u \in \mathscr{E}^{-1}\left(C_{0}^{\infty}\left(\mathbb{R}^{n}-\{0\}\right)\right)$ and $B$ a bounded set of $\mathbb{V}$. Then there exists a constant $T=T(\varepsilon, u, B)$ such that $\|W(t: V) u-W(V) u\| \leq \varepsilon$ holds for all $t \geq T$ and $V \in B$.

The proof of the above lemma can be obtained by a careful examination of the above cited authors' proof of Theorem 2.3, though they do not state explicit dependence on $V$ various estimates concerned.

Lemma 2.5. Let $V_{m} \rightarrow V$ in $\mathbb{V}$. Then for an arbitrary fixed $t$ $W\left(t: V_{m}\right) \rightarrow W(t: V)$ strongly in $L_{2}\left(\mathbb{R}^{n}\right)$ as $m \rightarrow \infty$.

Proof. In view of Lemma 2.2, we have by Lebesgue's dominated 
convergence theorem $e^{-i t H_{0}-i X\left(p, t: V_{m}\right)} \rightarrow e^{-i t H_{0}-i X(p, t: V)}$ strongly in $L_{2}\left(\mathbb{R}^{n}\right)$. On the other hand, $V_{m} \rightarrow V$ in $\mathbb{V}$ shows that $V_{m}$ tends to $V$ in the operator norm in $L_{2}\left(\mathbb{R}^{n}\right)$. By the well-known theorem on the perturbation of semi-groups (see e.g. K. Yosida [14] p. 269), we can thus assert that $e^{i t H\left(V_{m}\right)}$ converges strongly to $e^{i t H(V)}$. These two facts show that

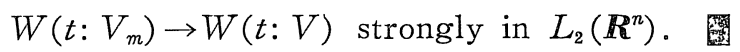

Theorem 2. 6. If $V_{m} \rightarrow V$ in $\mathbb{V}$, we have $W\left(V_{m}\right) \rightarrow W(V)$ strongly in $L_{2}\left(\mathbb{R}^{n}\right)$.

Proof. Since $W\left(V_{m}\right), W(V)$ are isometries (Theorem 2. 3), we have only to show that $W\left(V_{m}\right) u \rightarrow W(V) u$ in $L_{2}\left(\mathbb{R}^{n}\right)$ for $u$ in a dense set of $L_{2}\left(\mathbb{R}^{n}\right)$. Let $u \in \mathscr{F}^{-1}\left(C_{0}^{\infty}\left(\mathbb{R}^{n}-\{0\}\right)\right)$. By Lemma 2.4 , we see that for any $\varepsilon>0$ there exists a constant $T$ independent of $V_{m}$ such that $\| W(t$ : $\left.V_{m}\right) u-W\left(V_{m}\right) u\|+\| W(t: V) u-W(V) u \| \leq \varepsilon$ holds for all $t \geq T$. By Lemma 2.5, $W\left(T: V_{m}\right) u \rightarrow W(T: V) u$ in $L_{2}\left(\mathbb{R}^{n}\right)$ as $m \rightarrow \infty$. Finally, the inequality

$$
\begin{aligned}
\left\|W\left(V_{m}\right) u-W(V) u\right\| \leq & \left\|W\left(V_{m}\right) u-W\left(T: V_{m}\right) u\right\| \\
& +\left\|W\left(T: V_{m}\right) u-W(T: V) u\right\| \\
& +\|W(T: V) u-W(V) u\|
\end{aligned}
$$

shows that $W\left(V_{m}\right) u \rightarrow W(V) u$ in $L_{2}\left(\mathbb{R}^{n}\right)$. 娄

\section{§. Continuous Dependence of the Resolvents on $\mathbb{V}$}

To begin with, we introduce the following notations.

$$
\begin{aligned}
& \mathscr{D}_{j}=\mathscr{D}_{j}(z)=\frac{\partial}{\partial x_{j}}+\frac{n-1}{2 r} \widetilde{x}_{j}-i \sqrt{z} \widetilde{x}_{j}, \widetilde{x}_{j}=x_{j} / r, r=|x|, \operatorname{Im} \sqrt{ } z>0 .^{1)} \\
& \mathscr{D}=\mathscr{D}(z)=\left(\mathscr{D}_{1}, \cdots, \mathscr{D}_{n}\right) .
\end{aligned}
$$

For a domain $G$ in $\boldsymbol{R}^{n}$ and a real constant $\beta$, we define $L_{2, \beta}(G)$ as the Hilbert space of all measurable functions $f$ such that $\|f\|_{\beta, G}^{2}=\int_{G}(1+$

\footnotetext{
1) $\operatorname{Im}=$ imaginary part.
} 
$|x|)^{23}|f(x)|^{2} d x$ is finite. If $\beta=0$ or $G=\mathbb{R}^{n}$, we often omit the subscript.

$$
\begin{aligned}
& E_{R}=\left\{x \in \mathbb{R}^{n}:|x| \geq R\right\} . \\
& B_{R}=\left\{x \in \mathbb{R}^{n}:|x| \leq\{R .\right. \\
& B_{\rho, R}=\left\{x \in \mathbb{R}^{n}: \rho \leq|x| \leq R\right\} \quad(\rho<R) . \\
& R(z: V)=(H(V)-\approx)^{-1} \quad(z \in \mathbb{C}-\mathbb{R}) .{ }^{1)}
\end{aligned}
$$

$\mathbb{C}^{+}$and $\mathbb{C}^{-}$denote the upper and lower half plane in $\mathbb{C}$, respectively.

Let $D$ be an arbitrary bounded set in $\mathbb{C}-\mathbb{R}$ such that $\bar{D}$, the closure of $D$, has no intersection with $(-\infty, 0] . \quad H_{\mathrm{loc}}^{2}$ is the space of all measurable functions such that their distribution derivatives up to the second order are locally in $L_{2}$.

The following result has been established in Ikebe-Saitō [6].

Theorem 3.1 (Limiting Absorption Method). Let $V \in \mathbb{E}$ be fixed and $\varepsilon_{0}$ be a constant such that $0<\varepsilon_{0} \leq \delta / 2$. Then the following assertions hold.

(1) For any $f \in L_{2,\left(1+\varepsilon_{0}\right) / 2}$ and $\approx \in D, u=u(z: f: V)=R(z: V) f$ satisfies

$$
\begin{aligned}
& \|u(z: f: V)\|_{-\left(1+\varepsilon_{0}\right) / 2} \leq C\|f\|_{\left(1-\varepsilon_{0}\right) / 2}, \\
& \| \text { g) }(z) u(z: f: V)\left\|_{-\left(1-\varepsilon_{0}\right) / 2, E_{1}} \leq C\right\| f \|_{\left(1-\varepsilon_{0}\right) / 2},
\end{aligned}
$$

where the constant $C$ is independent of $\approx \in D, f \in L_{2,\left(1+\varepsilon_{0}\right) / 2}$.

(2) $u(z: f: V)$ is continuous in $L_{2,-\left(1, \varepsilon_{0}\right), 2}$ for $z \in D$ and $f \in L_{2,\left(1+\varepsilon_{0}\right) / 2}$, and, as a function of $z$, can be continuously extended in $L_{2,-\left(1+\varepsilon_{0}\right) / 2}$ to $\overline{D \cap C^{+}}$and $\overline{D \cap \mathbb{C}^{-}}$. The extended function $u(\lambda \pm i 0: f: V), \lambda \pm i 0 \in$ $\mathbb{R} \cap\left(\overline{D \cap \mathbb{C}^{x}}\right)$, satisfies the same estimates as (1) with $z=\lambda \pm i 0$.

(3) $u(\lambda \pm i 0: f: V)$ solves the following problem uniquely:

$$
\begin{aligned}
& (-J+V-\lambda) u=f, \quad f \in L_{2,\left(1+\varepsilon_{0}\right) / 2}, \\
& u \equiv L_{2,-\left(1, \varepsilon_{0}\right) / 2} \cap H_{\mathrm{loc}}^{\nu}, \\
& \mathscr{D}(\lambda \pm i 0) u \in L_{2,-\left(1-\varepsilon_{0}\right) / 2}\left(E_{1}^{\prime}\right) .
\end{aligned}
$$

Now, we want to investigate the dependence on $V$ of $u(\lambda \pm i 0: f: V)$. For this purpose we first prove the following

1) $C$ is the complex plane. 
Lemma 3.2. Let $V \in M$ a compact set in $V$. Then there exists a constant $C$ independent of $V \in M, f \in L_{2,\left(1+\varepsilon_{0}\right) / 2}$ and $z \in D \cap C^{+}$or $z \in D \cap \mathbb{C}^{-}$such that the following inequalities hold:

$$
\begin{aligned}
& \|u(z: f: V)\|_{-\left(1+\varepsilon_{0}\right) / 2} \leq C\|f\|_{\left(1+\varepsilon_{0}\right) / 2}, \\
& \|\mathscr{D}(z) u(z: f: V)\|_{-\left(1-\varepsilon_{0}\right) / 2, E_{1}} \leq C\|f\|_{\left(1+\varepsilon_{0}\right) / 2}, \\
& \|u(z: f: V)\|_{-\left(1+\varepsilon_{0}\right) / 2, E_{\rho}} \leq C \rho^{-\varepsilon_{0} / 2}\|f\|_{\left(1+\varepsilon_{0}\right) / 2}, \quad \rho>1 .
\end{aligned}
$$

Proof. ${ }^{1)}$ First we note that there exists a constant $C$ independent of $f \in L_{2,\left(1+\varepsilon_{0}\right) / 2}, z \in D$ and $V \in M$ such that the following inequalities hold:

$$
\begin{aligned}
& \|\mathscr{D}(z) u\|_{-\left(1-\varepsilon_{0}\right) / 2, E_{1}} \leq C\left\{\|u\|_{-\left(1+\varepsilon_{0}\right) / 2}+\|f\|_{\left(1+\varepsilon_{0}\right) / 2}\right\}, \\
& \|u\|_{-\left(1+\varepsilon_{0}\right) / 2, E_{\rho}}^{2} \leq C \rho^{-\varepsilon_{0}}\left\{\|u\|_{-\left(1+\varepsilon_{0}\right) / 2}^{2}+\|f\|_{\left(1+\varepsilon_{0}\right) / 2}^{2}\right\}, \quad \rho>1 .
\end{aligned}
$$

(3.1) and (3.2) have been shown in Lemmas 1.7 and 1.8 of IkebeSaito [6], though the dependence on $V$ of the constant $C$ has not been taken into account there. If we carefully reexamine the proof, however, we can find that $C$ in (3.1) and (3.2) is independent of $V$ in a bounded set in $\boldsymbol{V}$.

If (1) has been shown, (2) and (3) follow from (1) by using (3.1) and (3. 2). We shall prove (1). Suppose (1) is false. Then there exists a sequence $\left\{z_{m}, f_{m}, V_{m}\right\}_{m=1,2, \ldots}$ such that $\left\|u\left(z_{m}: f_{m}: V_{m}\right)\right\|_{-\left(1+\varepsilon_{0}\right) / 2}$ $=1,\left\|f_{m}\right\|_{\left(1+\varepsilon_{0}\right) / 2} \leq 1 / m$. Let us set $u_{m}=u\left(z_{m}: f_{m}: V_{m}\right)$ for brevity. Since $V_{m} \in M$, we can assume without loss of generality that $\approx_{n} \rightarrow z$ in $\overline{D \cap C^{+}}$ or $\overline{D \cap \mathbb{C}^{-}}$, and $V_{m} \rightarrow V$ in $V$ as $m \rightarrow \infty$. Let us show that there exists a function $v \in L_{2,-\left(1+\varepsilon_{0}\right) / 2} \cap H_{\text {loc }}^{2}$ having the following properties:

(3. 3) There exists a subsequence $\left\{u_{m^{\circ}}\right\}$ of $\left\{u_{m}\right\}$ such that

$$
u_{m^{\prime}} \rightarrow v \text { in } L_{2,-\left(1+\varepsilon_{0}\right) / 2} \text { as } m^{\prime} \rightarrow \infty \text {; }
$$

$$
\|\mathscr{D}(z) v\|_{-\left(1-\varepsilon_{0}\right) / 2, E_{1}}<\infty .
$$

In fact, by (3.1) and (3.2) we have

$$
\begin{aligned}
& \left\|u_{m}\right\|_{-\left(1+\varepsilon_{0}\right) / 2, E_{\rho}} \leq C \rho^{-\varepsilon_{0} / 2}, \quad \rho>1, \\
& \left\|\mathscr{D}\left(z_{m}\right) u_{m}\right\|_{-\left(1-\varepsilon_{0}\right) / 2, E_{1}} \leq C,
\end{aligned}
$$

1) Cf. the proof of Lemma 1.11 of [6]. 
where the constant $C$ is independent of $m$. By (3.5), for any $\varepsilon>0$, there exists a constant $R>1$ independent of $m$ such that for all $m$

$$
\left\|u_{m}\right\|_{-\left(1+\varepsilon_{0}\right) / 2, E_{R}}<\varepsilon .
$$

Recall the following well-known elliptic estimate which holds for $u \in H_{\text {loc }}^{2}$ and for $\rho<R$

$$
\begin{gathered}
\sum_{|\alpha| \leq 2} \int_{|x| \leq \rho}\left|D^{\alpha} u\right|^{2} d x \leq C(\rho, R)\left(\int_{|x| \leq R}|(-\Delta+V-z) u|^{2} d x\right. \\
\left.+\int_{|x| \leq R}|u|^{2} d x\right)
\end{gathered}
$$

where the constant $C(\rho, R)$ is independent of $z \in D$ and of $V$ if $\sup _{\mid x i<R}|V(x)|$ is uniformly bounded in $V$. In view of (3.8) $\left\|\nabla_{x} u_{m}\right\|_{B_{R}}+\left\|u_{m}\right\|_{B_{R}}$ is uniformly bounded in $m$. Hence by the usual diagonal process with (3.7) and Rellich's selection theorem in mind we see that there exist $v \in L_{2,-\left(1+\varepsilon_{0}\right) / 2}$ and a subsequence $\left\{u_{m^{\prime}}\right\}$ of $\left\{u_{m}\right\}$ such that $u_{m^{\prime}} \rightarrow v$ in

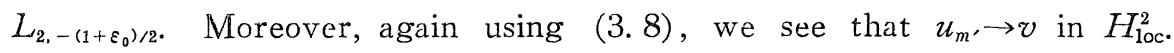
Let $R>1$. We have by (3. 6) $\left\|\mathscr{D}\left(z_{m^{\prime}}\right) u_{m^{\prime}}\right\|_{-\left(1-\varepsilon_{0}\right) / 2, B_{1, R}} \leq C$, where the constant $C$ is independent of $m^{\prime}$ and $R$. Letting $m^{\prime}$ tend to $\infty$ we have $\|\mathscr{D}(\approx) v\|_{-\left(1-\varepsilon_{0}\right) / 2, B_{1}, R} \leq C$. Letting $R$ tend to $\infty$ we have $\|\mathscr{D}(z) v\|_{-\left(1-\varepsilon_{0}\right) / 2, E_{1}}$ $<\infty$, so that we have proved the existence of $v \in L_{2,-\left(1+\varepsilon_{0}\right) / 2} \cap \mid H_{\mathrm{loc}}^{2}$ satisfying (3.3) and (3.4). As is easily seen $v$ satisfies $(-\Delta+V-z) v=0$, $v \in L_{2,-\left(1+\varepsilon_{0}\right) / 2}, \mathscr{D}(z) v \in L_{2,-\left(1-\varepsilon_{0}\right) / 2}\left(E_{1}\right)$. If $z=\lambda \pm i 0$ is real then the uniqueness result stated in Theorem 3.1 (3) shows $v=0$, which contradicts the fact that $\left\|u_{m},\right\|_{-\left(1+\varepsilon_{0}\right) / 2}=1$. If $z$ is non-real it is much easier to get to a contradiction.

Theorem 3. 3. Let $V_{m} \rightarrow V$ in $V$. We have for all $\lambda>0$ and $f \in L_{2,\left(1+\varepsilon_{0}\right) / 2}, R\left(\lambda \pm i 0: V_{m}\right) f \rightarrow R(\lambda \pm i 0: V) f$ in $L_{2,-\left(1+\varepsilon_{0}\right) / 2}$.

Proof. Set $u_{m}=R\left(\lambda \pm i 0: V_{m}\right) f$. In view of Lemma 3. 2 (3) there exists for an arbitrary $\varepsilon>0$ a constant $R$ independent of $m$ such that $\left\|u_{m}\right\|_{-\left(1+\varepsilon_{0}\right), 2, E_{R}}$ $<\varepsilon$. Keeping this in mind we can argue as in the proof of Lemma 3.2 to conclude that there exist a subsequence $\left\{u_{m^{\prime \prime}}\right\}$ of any subsequence $\left\{u_{m^{\prime}}\right\}$ of $\left\{u_{m}\right\}$ and $v \in L_{2,-\left(1+\varepsilon_{0}\right) / 2}$ such that $u_{m^{\prime \prime}} \rightarrow v$ in $L_{2,-\left(1+\varepsilon_{0}\right) / 2}$ and $v$ satisfies $(-1+V-\lambda) v=f$ and $\mathscr{D}(\lambda \pm i 0) v \in L_{2,-\left(1-\varepsilon_{0}\right) / 2}\left(E_{1}\right)$. But Theorem 
3.1 (3) shows that $v$ does not depend on the choice of the subsequence $\left\{u_{m^{\prime \prime}}\right\}$ of $\left\{u_{m^{\prime}}\right\}$. Since every subsequence $\left\{u_{m^{\prime}}\right\}$ of $\left\{u_{m}\right\}$ contains a subsequence $\left\{u_{m^{a}}\right\}$ which converges to one and the same limit, we see that the sequence $\left\{u_{m}\right\}$ itself converges, and the limit is just $R(\lambda \pm i 0: V) f$ by Theorem 3.1 (3).

\section{§4. Time-Dependent and Stationary Modifiers}

This section has a preliminary character to the following sections. We shall transform the time-dependent modifier introduced in Section 2 into a "stationary" modifier, which will enable us to develop in the next section an eigenfunction expansion theory.

First we note the following inverse function theorem.

Lemma 4. 1. Let $U$ be an open set in $\boldsymbol{R}^{N}$, and $\boldsymbol{R}_{+}=(0, \infty)$. Let $\left\{\Phi_{n}(p, \mu)\right\}_{n=1,2, \ldots}$ and $\Phi_{\infty}(p, l l)$ be $C^{\infty}\left(U \times \boldsymbol{R}_{-}\right)$-functions reith values in $\boldsymbol{R}^{N}$. We assume that $\Phi_{n}(p, \mu) \quad(n=1,2, \cdots ; \infty)$ have the following properties:

(1) $\Phi_{n}(p,, !)=p+\phi_{n}(p, !)$,

(2) $\sup _{n} \sup _{\mu \geq 1} \sup _{p \in \mathcal{U}}(1+\mu)^{m+\grave{o}}\left|D_{p}^{\alpha} D_{\mu}^{m} \phi_{n}(p, \mu)\right|<\infty$ for all $\alpha$ and $m(|\alpha| \geq 0$, $m \geq 0$ ),

(3) for all $c x$ and $m(|\alpha| \geq 0, m \geq 0)$ we have $D_{p}^{\alpha} D_{\mu}^{m} \phi_{n}(p, \iota) \rightarrow$ $D_{p}^{\alpha} D_{\mu}^{m} \phi_{\infty}(p, \mu)$ pointwise as $n \rightarrow \infty$.

Let $K$ be an arbitrary compact set in $U$. Then there exist $C^{\infty}\left(\boldsymbol{R}^{N}\right.$ $\left.\times \mathbb{R}_{+}\right)$-functions $\left\{\Psi_{n}(p, \mu)\right\}_{n=1,2, \ldots,}, \Psi_{\infty}(p, \mu)$ and a positive constant $R>1$ such that the following assertions hold:

(4) $\bar{\Phi}_{n}\left(\Psi_{n}(p, \mu), \mu\right)=p$ for $p \in K, \mu \geq R, n=1,2, \cdots ; \infty$,

(5) $\quad \sup _{n} \sup _{\mu \geq R} \sup _{p \in K}(1+\ell)^{m+\delta}\left|D_{p}^{\alpha} D_{\mu}^{m}\left(\Psi_{n}(p, \imath)-p\right)\right|<\infty$ for all $\alpha$ and $m$ $(|\alpha| \geq 0, m \geq 0)$,

(6) for $p \in K$ and $\mu \geq R$ ree have $D_{p}^{\alpha} D_{\mu}^{m} \Psi_{n}(p,, \iota) \rightarrow D_{p}^{\alpha} D_{\mu}^{m} \Psi_{\omega}(p, \iota)$ pointwise as $n \rightarrow \infty$ for all $\alpha$ and $m(|\alpha| \geq 0, m \geq 0)$,

(7) the function $\Psi_{n}(p, \mu)$ is unique in the sense that if $\Psi_{n}^{(1)}(p, \mu)$ and $\Psi_{n}^{(2)}(p, \mu)$ are such functions, we have $\Psi_{n}^{(1)}(p, l)=\Psi_{n}^{(2)}(p, \mu)$ for $p \in K$ and $\mu \geq R$. 
Proof. Since the proof is just the same as for the usual inverse function theorem, we only outline it. By our assumption (2), there exists a constant $R$ such that for $p \in U T, n \geq R$ and $n=1,2, \cdots ; \infty$,

$$
\operatorname{det}\left(1+D_{p} \phi_{n}(p, \mu)\right)>1 / 2 \text {. }
$$

where $D_{p} \phi_{n}(p, \mu)$ denotes the Jacobian matrix of $\phi_{n}(p, \mu)$ with respect to p. Moreover, we can also assume that

$$
\sup _{n} \sup _{\mu \geq R}\left|\phi_{n}(p, \ell)-\phi_{n}(q, \iota)\right| \leq|p-q| / 2 \text { for all } p, q \in U \text {. }
$$

Now, let us set $\Psi_{n}^{(0)}(p, \mu)=p, \Psi_{n}^{(j+1)}(p, \mu)=p-\phi_{n}\left(\Psi_{n}^{(j)}(p, \mu), \mu\right), j=0,1,2$, ... Taking / sufficiently large $(\ell \geq R)$ we have $\Psi_{n}^{(j)}(p, \mu) \in U$ when $p \in K$. Thus, this successive approximation scheme is well-defined. By (4. 2) we have for $p \in K$,,$\geq R\left|\Psi_{n}^{(j+1)}(p, \mu)-\Psi_{n}^{(j)}(p, \mu)\right| \leq \mid \Psi_{n}^{(j)}(p, \mu)$ $-\Psi_{n}^{(j-1)}(p, \ell) \mid / 2$. Hence we see that $\Psi_{n}^{(j)}(p, \ell)$ converges as $j \rightarrow \infty$ uniformly for $p \in K, \mu \geq R$ and $n$. Let us define $\Psi_{n}(p, \mu)=\lim _{j \rightarrow \infty} \Psi^{(j)}(p, \mu)$. It is easy to see that $\Psi_{n}(p, \mu)$ has the following properties:

$$
\Psi_{n}(p, \mu)=p-\phi_{n}\left(\Psi_{n}(p, \mu), \iota\right) \quad \text { for } p \in K, \mu \geq R,
$$

(4. 4) $\Psi_{n}\left(p,,^{\prime} \ell\right)$ is a continuous function of $p \in K$ and $\mu \geq R$,

and is uniformly bounded for $p \in K, \mu \geq R$ and $n$,

$$
T_{n}(p, \iota) \rightarrow \Psi_{\infty}(p, \mu) \text { as } n \rightarrow \infty \quad \text { for } p \in K, \mu \geq R \text {. }
$$

By (4.3) we have

$$
\Phi_{n}\left(\Psi_{n}(p, \mu), \mu\right)=p \quad \text { for } p \in K, \mu \geq R .
$$

By assumption (2) and (4.3) we have

$$
\sup _{n} \sup _{\mu \geq R} \sup _{p \in K}(1+\ell)^{\delta}\left|\Psi_{n}(p, \mu)-p\right|<\infty .
$$

We can see by (4.3) and an elementary argument that $\Psi_{n}(p, \mu)$ is differentiable with respect to $p$ and satisfies $D_{p} \Psi_{n}(p, l)=1-\left(D_{p} \phi_{n}\right)\left(\Psi_{n}(p, \mu)\right.$,

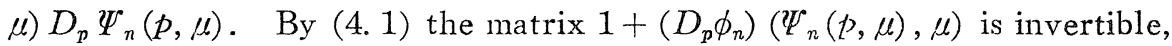
hence we have

$$
\begin{aligned}
D_{p}\left(\Psi_{n}(p, \mu)-p\right)= & -\left(1+\left(D_{p} \phi_{n}\right)\left(\Psi_{n}(p, \mu), \mu\right)\right)^{-1} \\
& \times\left(D_{p} \phi_{n}\right)\left(\Psi_{n}(p, \mu), \ell\right) .
\end{aligned}
$$

In view of assumption (2) and (4.8), we can conclude 


$$
\sup \sup _{\mu \geq R} \sup _{p \in K}(1+\mu)^{\delta} \mid D_{p}\left(\Psi_{n}(p, \mu)-p\right) i<\infty,
$$

which proves (5) when $|\alpha|=1, m=0$. Also by assumption (3), (4.5) and (4.8)

$$
D_{p}\left(\Psi_{n}(p, \mu)-\Psi_{\infty}(p, \mu)\right) \rightarrow 0 \quad \text { as } n \rightarrow \infty,
$$

which proves (6) when $|\alpha|=1, m=0$. Again by (4.3) and an elementary argument we can see that $\Psi_{n}(p, \mu)$ is differentiable with respect to $\mu$ and satisfies

$$
D_{\mu} \Psi_{n}(p, \mu)=-\left(D_{p} \phi_{n}\right)\left(\Psi_{n}(p, \mu), \mu\right) D_{\mu} \Psi_{n}(p, \mu)-\left(D_{\mu} \phi_{n}\right)\left(\Psi_{n}(p, \mu), \mu\right) .
$$

Hence we have

$$
\begin{aligned}
D_{\mu} \Psi_{n}(p, \mu)= & -\left(1+\left(D_{p} \phi_{n}\right)\left(\Psi_{n}(p, \mu), \mu\right)\right)^{-1} \\
& \times\left(D_{\mu} \phi_{n}\right)\left(\Psi_{n}(p, \mu), \mu\right) .
\end{aligned}
$$

In view of (2) and (4.11) we get

$$
\sup \sup _{\mu \geq R} \sup _{p \in K}(1+\mu)^{1+\grave{o}}\left|D_{\mu} \Psi_{n}(p, \mu)\right|<\infty,
$$

which proves (5) when $|\alpha|=0$ and $m=1$. We can further see by (3), (4.5) and (4.11)

$$
D_{\mu}\left(\Psi_{n}(p, \mu)-\Psi_{\infty}(p, \mu)\right) \rightarrow 0 \quad \text { as } n \rightarrow \infty,
$$

which proves (6) when $|\alpha|=0, m=1$. Again in view of (4.8) and (4.11) $\Psi_{n}(p, \mu)$ is a $C^{\infty}$-function of $p \in K$ and $\mu \geq R$. The assertions (5) and (6) follow thus by induction. For $p \notin K$ or $\mu<R$, we continue $\Psi_{n}(p, \mu)$ in a $C^{\infty}$-fashion. The uniqueness assertion (7) can be shown by (4.2).

Now let us define

$$
W(\xi, t: V)=t|\xi|^{2}+X(\xi, t: V),
$$

and consider the equations

$$
x=\frac{\partial W^{1)}}{\partial \xi}, \lambda=\frac{\partial W}{\partial t} .
$$

Using these equations we want to express $\xi$ and $t$ as functions of $x$ and $\lambda$, which is made possible by the following lemma.

1) $\frac{\partial}{\partial \xi}=\nabla_{\xi}$. 
Lemma 4.2. Let $X(\xi, t: V)$ be a time-dependent modifier. Then there exist $C^{\infty}\left(\mathbb{R}^{n} \times \mathbb{R}_{+}\right)$-functions $\xi(x, \lambda)=\xi(x, \lambda: V), t(x, \lambda)$ $=t(x, \lambda: V)$ having the following properties: If $\Lambda$ is a compact set in $\mathbb{R}_{+}$, then there exists a constant $R$ such that

$$
x=2 \xi(x, \lambda) t(x, \lambda)+\left(\nabla_{\xi} X\right)(\xi(x, \lambda), t(x, \lambda): V),
$$

$$
\text { for }|x| \geq R, \lambda \in \Lambda \text {, }
$$

(2) $\lambda=|\xi(x, \lambda)|^{2}+\left(\frac{\partial X}{\partial t}\right)(\xi(x, \lambda), t(x, \lambda): V)$, for $|x| \geq R, \lambda \in \mathbb{A}$,

where the constant $R$ is independent of $T^{\top}$ if $V$ varies over a bounded set in $\boldsymbol{V}$. If we set

$$
\begin{aligned}
& \phi(x, \lambda)=t(x, \lambda)-\frac{r}{2 \sqrt{\lambda}}, \\
& \psi(x, \lambda)=\xi(x, \lambda)-\sqrt{\lambda}(\omega,
\end{aligned}
$$

where $r=|x|, \omega=x / r$, then we have for every $\alpha$ and $m(|\alpha| \geq 0, m \geq 0)$

$$
\begin{aligned}
& \left|D_{x}^{\alpha} D_{\lambda}^{m} \phi(x, \lambda)\right| \leq C(1+|x|)^{1-|\alpha|-\hat{o}}, \\
& \left|D_{x}^{\alpha} D_{\lambda}^{m} \psi(x, \lambda)\right| \leq C(1+|x|)^{-|\alpha|-\hat{o}}, \quad(|x|>1, \lambda \in \Lambda),
\end{aligned}
$$

where the constant $C$ is independent of $\lambda \in \Lambda$ and $V$ in a bounded set of $\mathbb{V}$.

Proof. First we rewrite the equations $x=\frac{\partial W}{\partial \xi}, \lambda=\frac{\partial W}{\partial t}$ as follows:

$$
\begin{aligned}
& \omega=2 \xi \frac{t}{r}+\frac{1}{r}\left(\nabla_{\xi} X\right)(\xi, t ; V) \quad(r=|x|, \omega=x / r), \\
& \lambda=|\xi|^{2}+\left(\frac{\partial X}{\partial t}\right)(\xi, t: V) .
\end{aligned}
$$

Let us introduce new variables $\zeta=2 \xi t / r, s=|\xi|^{2}$. Then we have

$$
\begin{aligned}
& \omega=\zeta+\frac{1}{r}\left(\nabla_{\xi} X\right)\left(\sqrt{s} \frac{\zeta}{|\zeta|}, \frac{r|\zeta|}{2 \sqrt{s}}: V\right), \\
& \lambda=s+\left(\frac{\partial X}{\partial t}\right)\left(\sqrt{s} \frac{\zeta}{|\zeta|}, \frac{r|\zeta|}{2 \sqrt{s}}: V\right) .
\end{aligned}
$$

The function 


$$
\Phi(\zeta, s, r)=\left(\zeta+\frac{1}{r}\left(\nabla_{\xi} X\right)\left(\sqrt{s} \frac{\zeta}{|\zeta|}, \frac{r|\zeta|}{2 \sqrt{s}}: V\right), s+\left(\frac{\partial X}{\partial t}\right)\left(\sqrt{s} \frac{\zeta}{|\zeta|}, \frac{r|\zeta|}{2 \sqrt{s}}: V\right)\right)
$$

defines a $C^{\infty}$-map of $(\zeta, s)$ and $r(\zeta \neq 0, s>0, r>0)$. Let $K$ be a compact set in $\mathbb{R}^{n}-\{0\}$ containing the unit sphere and $\Lambda$ be a compact set in $\boldsymbol{R}_{+}$. By Lemma 2.2, we see that $\Phi(\zeta, s, r)$ satisfies the conditions (1), (2) and (3) of Lemma 4. 1, where $p=(\zeta, s), \mu=r$ and $\Phi_{n}(p, \mu)=\Phi((\zeta, s), r)$ for all $n=1,2, \cdots ; \infty, K=K \times \Lambda$ and we take for $U$ an open set contain. ing $K \times A$ bounded away from $(0,0)$. Therefore, there exist $C^{\infty}$-functions $\zeta(\omega, \lambda, r)$ and $s(\omega, \lambda, r)$ satisfying (4.15) for $(\omega, \lambda) \in K \times \Lambda$ and for sufficiently large $r$. We set

$$
\begin{aligned}
& \xi(x, \lambda)=\sqrt{s((1), \lambda, r) \zeta(\omega, \lambda, r) /|\zeta(\omega, \lambda, r)|,} \\
& t(x, \lambda)=r|\zeta(\omega, \lambda, r)| /(2 \sqrt{s(\omega, \lambda, r)}),
\end{aligned}
$$

where $r=|x|, \omega=x / r$. These functions $\xi(x, \lambda), t(x, \lambda)$ satisfy (1) and (2) for $|x| \geq R$ ( $R$ sufficiently large) and $\lambda \in \Lambda$. The estimates (3) follow from the estimates (5) of Lemma 4.1.

Next let us choose a sequence of compact sets $\Lambda_{j} \subset \boldsymbol{R}_{\perp}$ such that $\Lambda_{1} \subset \Lambda_{2} \subset \cdots \rightarrow \mathbb{R}_{+}$. For each $\Lambda_{j}$ we can construct $\xi^{(j)}(x, \lambda)$ and $t^{(j)}(x, \lambda)$ having the properties (1), (2) and (3) with $R=R_{j}$. Without loss of generality we can assume that $R_{1}<R_{2}<\cdots \rightarrow \infty$. Then the uniqueness result of Lemma 4.1 shows that if $j \geq k \xi^{(j)}(x, \lambda)=\xi^{(k)}(x, \lambda), t^{(j)}(x, \lambda)$ $=t^{(k)}(x, \lambda)$, for $(x, \lambda) \in\left\{x:|x| \geq R_{j}\right\} \times \Lambda_{k}$. We re-define $\xi^{(j)}(x, \lambda)$ and $t^{(j)}(x, \lambda)$ by $\xi^{(j)}(x, \lambda)=\xi^{(k)}(x, \lambda), t^{(j)}(x, \lambda)=t^{(k)}(x, \lambda)$ for $(x, \lambda) \in\left\{x: R_{k}\right.$ $\left.\leq|x| \leq R_{j}\right\} \times \Lambda_{k}$.

Let $A=\cup\left\{x:|x| \geq R_{k}\right\} \times \Lambda_{k}$. then the functions $\xi_{0}(x, \lambda)=\xi^{(k)}(x, \lambda)$, $t_{0}(x, \lambda)=t^{(k)}(x, \lambda)$ for $(x, \lambda) \in\left\{x:|x| \geq R_{k}\right\} \times A_{k}$ are well-defined on $A$. Choose numbers $\widetilde{R}_{j}$ and compact sets $\widetilde{\Lambda}_{j}$ so that $\widetilde{R}_{j}>R_{j}, \widetilde{\Lambda}_{j} \subset \Lambda_{j}, \widetilde{\Lambda}_{1} \subset \tilde{\Lambda}_{2}$ $\subset \cdots \rightarrow \boldsymbol{R}_{+}$. Define $\tilde{A}=\bigcup\left\{x:|x| \geq \widetilde{R}_{k}\right\} \times \widetilde{\Lambda}_{k} \subset A$. Then there exists a real $C^{\infty}\left(\mathbb{R}^{n} \times \mathbb{R}_{+}\right)$-function $\phi(x, \lambda)$ such that $\phi(x, \lambda)=1$ for $(x, \lambda) \in \tilde{A}$ and $\phi(x, \lambda)=0$ for $(x, \lambda) \notin A$. Finally we define $\xi(x, \lambda)$ and $t(x, \lambda)$ by $\xi(x, \lambda)=\phi(x, \lambda) \xi_{0}(x, \lambda)$ for $(x, \lambda) \in A, 0$ otherwise; $t(x, \lambda)=\phi(x, \lambda)$ $t_{0}(x, \lambda)$ for $(x, \lambda) \in A, 0$ otherwise. Then we see that $\xi(x, \lambda)$ and $t(x, \lambda)$ have all the desired properties.

The following lemma will be employed in Section 7 . 
Lemma 4. 3. If $V \subseteq V$ has a compact support, we have the following asymptotic estimate for $r \rightarrow \infty$

$$
\begin{aligned}
& t(x, \lambda: V)=r /(2 \sqrt{\lambda})+O(1), \\
& \xi(x, \lambda: V)=\sqrt{\lambda}(1)+O\left(r^{-1}\right), \quad r=|x|, \quad \omega=x / r .
\end{aligned}
$$

Proof. Let $A\left(\xi, t: T^{\top}\right)=\frac{\partial}{\partial t} X(\xi, t: V)-V\left(2 \xi t+\nabla_{\xi} X(\xi, t: V)\right)$.

Then we have

$$
\begin{aligned}
X\left(\hat{\xi}, t: V^{\top}\right)=X\left(\xi, 1: V^{\top}\right)+\int_{1}^{t}\left[T^{\top}(2 \xi s\right. & \left.+\nabla_{\xi} X\left(\xi, s: V^{\top}\right)\right) \\
& +A(\xi, s: V)] d s .
\end{aligned}
$$

By Lemma 2.2 we have for $\xi \neq 0$

$$
\left|D_{\xi}^{\alpha} A\left(\xi, t: T^{\top}\right)\right| \leq C(1+t)^{-2}, \quad|\alpha|=0,1 .
$$

Taking into account that $V$ has a compact support, we have the following estimates: For $t>1, \xi \neq 0$,

$$
\begin{aligned}
& \left|\nabla_{\xi} X(\xi, t: V)\right| \leq C\left(1+\int_{1}^{t}(1+s)^{-2} d s\right) . \\
& \left|\frac{\partial}{\partial t} X(\xi, t: V)\right| \leq C(1+t)^{-2} .
\end{aligned}
$$

We are thus led, in view of (4.15), to asymptotic relations $\zeta(\omega, \lambda, r)$ $=\omega+O\left(r^{-1}\right), s(\omega, \lambda . r)=\lambda+O\left(r^{-2}\right)$, which give rise to

$$
\begin{aligned}
& t(x, \lambda: V)=r|\zeta(\omega, \lambda, r)| /(2 \sqrt{s(\omega, \lambda, r)}) \\
& =r\left|\omega+O\left(r^{-1}\right)\right| /\left(2\left(\sqrt{ } \lambda+O\left(r^{-2}\right)\right)\right) \\
& =r /(2 \sqrt{ } \lambda)+O(1), \\
& \xi(x, \lambda: V)=\sqrt{s(\omega, \lambda, r)} \zeta(\omega, \lambda, r) /|\zeta(\omega, \lambda, r)| \\
& =\left(\sqrt{ } \bar{\lambda}+O\left(r^{-2}\right)\right)\left(\omega+O\left(r^{-1}\right)\right) /\left|\omega+O\left(r^{-1}\right)\right| \\
& =\sqrt{\lambda} \omega+O\left(r^{-1}\right) \text {. }
\end{aligned}
$$

Lemma 4. 4. Let $V_{n} \rightarrow V$ in $\boldsymbol{V}$. Then for all $\alpha$ and $m$ we have for $n \rightarrow \infty D_{x}^{\alpha} D_{\lambda}^{m}\left(t\left(x, \lambda: V_{n}\right)-t\left(x, \lambda: V^{r}\right)\right) \rightarrow 0, I_{x}^{a} D_{l}^{m}\left(\xi\left(x, \lambda: V_{n}\right)-\xi(x, \lambda:\right.$ V) $\rightarrow$ ) 
Proof. If we let in Lemma $4.1 \Phi_{n}(p, \mu)=\Phi\left(\zeta, s, r: V_{n}\right) \quad(p=(\zeta, s)$, $\mu=r)$, then the conditions of Lemma 4.1 are fulfilled. Reviewing the proof of Lemma 4.2 with condition (3) and assertion (6) of Lemma 4.1 in regard yields the conclusion of the lemma.

Now, we are to produce the stationary modifier $Y(x, \lambda: V)$ from the time-dependent modifier $X(\xi, t: V)$. Let $X(\xi, t: V)$ be a time-dependent modifier defined in Section 2 , and $\xi(x, \lambda: V), t(x, \lambda: V)$ as above. Choose a sequence of compact sets $\Lambda_{1} \subset \Lambda_{2} \subset \cdots \rightarrow \mathbb{R}_{+}$. By Lemma 4.2 (3), we find for each $j$ a constant $R_{j}$ such that $\xi(x, \lambda: V) \neq 0, t(x, \lambda: V)>0$ for $|x| \geq R_{j}, \lambda \in \Lambda_{j}$. Let $A=\cup_{j}\left\{x:|x| \geq R_{j}\right\} \times \Lambda_{j}$. Let $\hat{A}$ be a neighbourhood of $A$ contained in $\mathbb{R}^{n} \times \mathbb{R}_{+}$in which $\xi(x, \lambda: V) \neq 0, t(x, \lambda: V)>0$ still hold. Let $\phi(x, \lambda)$ be a real $C^{\infty}\left(\mathbb{R}^{n} \times \boldsymbol{R}_{+}\right)$-function such that $\phi(x, \lambda)=1$ on $A$ and $=0$ outside $\hat{A}$.

Lemma 4. 5. Let $\phi(x, \lambda)$ be as above. Let for $x \in \mathbb{R}^{n}, \lambda \in \mathbb{R}_{+}$, $V \in V$

$$
\begin{aligned}
K(x, \lambda: V)= & \phi(x, \lambda)[W(\xi(x, \lambda: V), t(x, \lambda: V): V) \\
& -x \xi(x, \lambda: V)-\lambda t(x, \lambda: V)],
\end{aligned}
$$

where $W(\xi, t: V)$ has been defined by (4.14). Then $K(x, \lambda: V)$ is a real $C^{\infty}\left(\mathbb{R}^{n} \times \mathbb{R}_{+}\right)$-function verifying the inequality

$$
\left.|| \nabla_{x} K(x, \lambda: V)\right|^{2}-\lambda+V(x) \mid \leq C(1+|x|)^{-2},
$$

where the constant $C$ is independent of $\lambda$ and $V$ if they vary over a compact set in $\mathbb{R}_{+}$and a bounded set in $\mathbb{V}$, respectively.

Proof. Since by Lemma 2. 2, W( $\xi, t: V)$ is a $C^{\infty}$-function of $\xi \neq 0$ and $t>0, K(x, \lambda: V)$ is smooth for $x \in \mathbb{R}^{n}$ and $\lambda>0$. Let $\Lambda$ be a compact set in $\boldsymbol{R}_{+}$. Then by Lemma 4.2 there exists a constant $R>0$ such that for $|x| \geq R, \lambda \in \Lambda$

$$
\begin{aligned}
& x=\frac{\partial W}{\partial \xi}(\xi(x, \lambda: V), t(x, \lambda: V): V), \\
& \lambda=\frac{\partial W}{\partial t}(\xi(x, \lambda: V), t(x, \lambda: V): V) .
\end{aligned}
$$


Direct calculation shows that for $|x| \geq R, \lambda \in \Lambda$

$$
\begin{aligned}
\frac{\partial}{\partial x_{j}} K(x, \lambda: V) & =\sum_{k} \frac{\partial W}{\partial \xi_{k}} \frac{\partial \xi_{k}}{\partial x_{j}}+\frac{\partial W}{\partial t} \frac{\partial t}{\partial x_{j}}-\xi_{j}-\sum_{k} x_{k} \frac{\partial \xi_{k}}{\partial x_{j}}-\lambda \frac{\partial t}{\partial x_{j}} \\
& =-\xi_{j} \quad(\text { by }(4.19)) .
\end{aligned}
$$

That is, for $|x| \geq R$ and $\lambda \in \Lambda$

$$
-\xi(x, \lambda: V)=\frac{\partial K}{\partial x}(x, \lambda: V) .
$$

On the other hand, Lemma 2.2 and (4.14) show

$$
\frac{\partial W}{\partial t}(\xi, t: V)-|\xi|^{2}-V\left(\frac{\partial W}{\partial \xi}(\xi, t: V)\right), \leq C(1+t)^{-2}
$$

In view of (4.19) and (4. 20) we have for $|x| \geq R, \lambda \in \Lambda$

$$
\begin{gathered}
\frac{\partial W}{\partial t}(\xi, t: V)-|\xi|^{2}-V\left(\frac{\partial W}{\partial \xi}(\xi, t: V)\right) \\
=\lambda-\left|\nabla_{x} K(x, \lambda: V)\right|^{2}-V(x) .
\end{gathered}
$$

This together with (4.21) and Lemma 4.2 (3) shows (4.18). 溉

Definition 4. 6. Let $\phi(x)$ be a real $C^{\infty}$-function such that $\phi(x)=0$ for $|x| \leq 1$ and $\phi(x)=1$ for $|x| \geq 2$. Define $Y(x, \lambda: V)$ by

$$
Y(x, \lambda: V)=\phi(x)(\sqrt{\lambda} r+K(x, \lambda: V)), \quad|x|=r .
$$

We shall call $Y(x, \lambda: Y)$ a stationary modifier associated with $V$.

Lemma 4. 7. $Y(x, \lambda: V)$ is a real $C^{\infty}\left(\mathbb{R}^{n} \times \mathbb{R}_{+}\right)$-function of $x$ and $\lambda$ having the following properties:

$$
\begin{aligned}
& \left|D_{x}^{\alpha} D_{\lambda}^{m}(x, \lambda: V)\right| \leq C(1+|x|)^{1-|\alpha|-\delta} \quad(|\alpha| \geq 0, m \geq 0), \\
& \left.\left|2 \sqrt{\lambda} \frac{\partial Y}{\partial r}(x, \lambda: V)-\right| \nabla_{x} Y(x, \lambda: V)\right|^{2}-V(x) \mid \leq C(1+|x|)^{-1},
\end{aligned}
$$

where the constant $C$ is independent of $\lambda$ and $V$ if they vary over $a$ compact set in $\mathbb{R}_{+}$and a bounded sei in $\mathbb{V}$, respectively,

(4) if $V_{n} \rightarrow T$ in $\mathbb{V}$, then re have for all $\alpha$ and $m D_{x}^{\alpha} D_{i}^{m} Y\left(x, \lambda: V_{n}\right)$ $\rightarrow D_{x}^{\alpha} D_{\lambda}^{m} Y(x, \lambda: V)$ as $n \rightarrow \infty$. 
Proof. Putting $\phi(x, \lambda: V)=t(x, \lambda: V)-r /(2 \sqrt{\lambda}), \quad \psi(x, \lambda: V)=$ $\xi(x, \lambda: V)-\sqrt{\lambda} \omega(r=|x|, \omega=x / r)$, one can rewrite $Y(x, \lambda: V)$ as

$$
\begin{aligned}
Y(x, \lambda: V)=\phi(x, \lambda) & {\left[X(\xi(x, \lambda: V), t(x, \lambda: V): V)+\frac{r}{2 \sqrt{\lambda}}|\psi(x, \lambda: V)|^{2}\right.} \\
& +2 \sqrt{\lambda} \omega \phi(x, \lambda: V) \phi(x, \lambda: V) \\
+ & \left.\phi(x, \lambda: V)|\psi(x, \lambda: V)|^{2}\right] .
\end{aligned}
$$

Then (1) follows from Lemmas 2.2 and 4.2. By direct calculation we have for $|x|>2$

$$
\left|\nabla_{x} K\right|^{2}-\lambda+V(x)=\left|\nabla_{x} Y\right|^{2}+V(x)-2 \sqrt{\lambda} \frac{\partial Y}{\partial r} .
$$

which combined with Lemma 4.5 proves (2). (3) is direct from Definition 4.6. Finally (4) follows from Lemmas 2.2 and 4. 4.

Remark 4. 8. The function $W(\xi, t: V)=t|\xi|^{2}+X(\xi, t: V)$ possesses, according to Lemma 2.2, an asymptotic estimate $\partial W / \partial t-|\xi|^{2}-$ $V(\partial W / \partial \xi)=O\left(t^{-2}\right)$, which means that $W(\xi, t: V)$ is an approximate solution of the "Hamilton-Jacobi" equation

$$
\frac{\partial W}{\partial t}=H\left(\frac{\partial W}{\partial \xi}, \xi\right)=|\xi|^{2}+V\left(\frac{\partial W}{\partial \xi}\right)
$$

where $H(x, \xi)$ is the classical Hamiltonian $H(x, \xi)=|\xi|^{2}+V(x)$. The variables $\xi$ and $t$ have the meaning of momentum and time. What we have done in Lemma 4.2 is to transfer from $\xi, t$ to the variables $x, \lambda$, which are the position and energy conjugate to the momentum and time, by means of the "Legendre transformation" $x=\partial W / \partial \xi, \lambda=\partial W / \partial t$. The "Legendre transform" of the function $W(\xi, t: V)$ is $K(x, \lambda: V)=W(\xi, t: V)$ $-x \xi-\lambda t$, and this function $K(x, \lambda: V)$ asymptotically satisfies the "eikonal equation"

$$
H\left(x, \frac{\partial K}{\partial x}\right)=\lambda \quad \text { or } \quad\left|\frac{\partial K}{\partial x}\right|^{2}=\lambda-V(x) .
$$

The stationary modifier $Y(x, \lambda: V)$ is to the "eikonal" $K(x, \lambda: V)$ what the time-dependent modifier $X(\xi, t: V)$ is to the "Hamilton-Jacobi" solution $W(\xi, \lambda: V)$. Kitada $[10]$, too, has observed the essentially same re- 
lation between the time-dependent and stationary modifiers from a slightly different point of view.

\section{§ 5. Eigenfunction Expansions and Stationary Wave Operators}

We start with defining a stationary wave operator investigated in Isozaki [7]. Let $R_{0}(z)$ denote the resolvent of $H_{0}=-\Delta$, and let $E_{0}(\lambda)$, $E(\lambda: V)$ be the resolutions of the identity for $H_{0}$ and $H(V)$, respectively. $\mathbb{B}\left(\mathcal{K}_{1}: \mathscr{K}_{2}\right)$ will denote the totality of bounded operators from a Banach space $\mathscr{H}_{1}$ to a Banach space $\mathscr{H}_{2}$.

Definition 5. 1. For $\lambda>0$ let $E_{0}^{\prime}(\lambda)$ and $E^{\prime}(\lambda: V)$ be defined by

$$
\begin{aligned}
& E_{0}^{\prime}(\lambda)=\frac{1}{2 \pi i}\left[R_{0}(\lambda+i 0)-R_{0}(\lambda-i 0)\right], \\
& E^{\prime}(\lambda: V)=\frac{1}{2 \pi i}[R(\lambda+i 0: V)-R(\lambda-i 0: V)],
\end{aligned}
$$

where we notice by Theorem 3.1 that $E_{0}^{\prime}(\lambda), E^{\prime}(\lambda: V) \in \mathbb{B}\left(L_{2,\left(1+\varepsilon_{0}\right) / 2}\right.$ : $\left.L_{2,-\left(1+\varepsilon_{0}\right) / 2}\right)$.

Definition 5.2. Let $U(z: V)$ be the unitary operator of multiplication by the function $\exp \left(-i Y\left(x,(\operatorname{Re} \sqrt{z})^{2}: V\right)\right)^{1)}$, where $Y(x, \lambda: V)$ $i s$ a stationary modifier (Definition 4.6). We define for $\operatorname{Im} z \neq 0$

$$
\begin{aligned}
& G(z: V)=\left(H_{0}-z\right) U(z: V) * R(z: V), \\
& \widetilde{G}(z: V)=(H(V)-z) U(z: V) R_{0}(z) .
\end{aligned}
$$

The following lemma gives a basis of our subsequent arguments.

Lemma 5. 3. (1) For $\lambda>0$, there exist strong limits $\operatorname{s}_{\varepsilon \downarrow 0} \lim (\lambda$ $+i \varepsilon: V) \equiv G(\lambda+i 0: V), \operatorname{s}_{\varepsilon \downarrow 0} \lim \widetilde{G}(\lambda+i \varepsilon: V) \equiv \widetilde{G}(\lambda+i 0: V)$ in $\mathbb{B}\left(L_{2,\left(8-\varepsilon_{0}\right), 2}:\right.$ $\left.L_{2,\left(1+\varepsilon_{0}\right) / 2}\right)$.

(2) For an arbitrary $f \in L_{2,\left(3-\varepsilon_{0}\right) / 2}, G(\lambda+i 0: V) f$ and $\widetilde{G}(\lambda+i 0: V) f$ are strongly continuous functions of $\lambda>0$.

1) $\operatorname{Re}=$ real part. 
For the proof see the proof of Isozaki [7] Theorem 3. Note that all we need about $Y(x, \lambda: V)$ to follow Isozaki's arguments are already stated in Lemma 4.7.

By Definition 5. 1 and Lemma 5. 3 it follows that the following definition makes sense.

Definition 5. 4. For $f \in L_{2,\left(3-\varepsilon_{0}\right) / 2}$ and $0<a<b<\infty$ we define

$$
\begin{aligned}
& \Gamma\left((a, b): H(V): H_{0}\right) f=\int_{a}^{b} E^{\prime}(\lambda: V) \widetilde{G}(\lambda+i 0: V) f d \lambda, \\
& \Gamma\left((a, b) ; H_{0}: H(V)\right) f=\int_{a}^{b} E_{0}^{\prime}(\lambda) G(\lambda+i 0: V) f d \lambda .
\end{aligned}
$$

It is easy to see by virtue of Theorem 3.1 that $\Gamma\left((a, b): H(V): H_{0}\right)$, $\Gamma\left((a, b): H_{0}: H(V)\right) \in B\left(L_{2,\left(3-\varepsilon_{0}\right) / 2}: L_{2,-\left(1+\varepsilon_{0}\right) / 2}\right)$.

Theorem 5. 5. (1) The operators $\Gamma\left((a, b): H(V): H_{0}\right)$ and $\Gamma\left((a, b): H_{0}: H(V)\right)$ actually map into $\mathcal{H}=L_{2}\left(\mathbb{R}^{n}\right)$ and for $f \in L_{2,\left(3-\varepsilon_{0}\right) / 2}$,

$$
\begin{aligned}
& \left\|\Gamma\left((a, b): H(V): H_{0}\right) f\right\|=\left\|E_{0}((a, b)) f\right\|, \\
& \left\|\Gamma\left((a, b): H_{0}: H(V)\right) f\right\|=\|E((a, b): V) f\| .
\end{aligned}
$$

(2) For $f \in L_{2,\left(3-\varepsilon_{0}\right) / 2}$ strong limits

$$
\begin{aligned}
& \Gamma\left(H(V): H_{0}\right) f \equiv \operatorname{sim}_{\substack{b \rightarrow \infty \\
a \rightarrow 0}} \Gamma\left((a, b): H(V): H_{0}\right) f, \\
& \Gamma\left(H_{0}: H(V)\right) f \equiv \operatorname{sim}_{\substack{b \rightarrow \infty \\
a \rightarrow 0}} \Gamma\left((a, b): H_{0}: H(V)\right) f
\end{aligned}
$$

exist in $\mathcal{H}=L_{2}\left(\boldsymbol{R}^{n}\right) . \quad \Gamma\left(H(V): H_{0}\right) \quad\left(\Gamma\left(H_{0}: H(V)\right)\right)$ is uniquely extended to a partial isometry on $\mathcal{H}$ with initial set $\mathcal{H}\left(\mathcal{H}_{a c}(H(V))\right)$ and final set $\mathcal{H}_{a c}(H(V))(\mathcal{H})$. We use the same notation for this extension.

(3) $\Gamma\left(H(V): H_{0}\right) *=\Gamma\left(H_{0}: H(V)\right), \quad \Gamma\left(H_{0}: H(V)\right) *=\Gamma\left(H(V): H_{0}\right)$, where $*$ denotes the adjoint in $\mathcal{H}$.

(4) The following intertwining property holds:

$$
\begin{aligned}
& H(V) \Gamma\left(H(V): H_{0}\right) \supseteq \Gamma\left(H(V): H_{0}\right) H_{0}, \\
& H_{0} \Gamma\left(H_{0}: H(V)\right) \supseteq \Gamma\left(H_{0}: H(V)\right) H(V) .
\end{aligned}
$$


For the proof see Theorems 1 and 3 of Isozaki [7].

Now we turn to the eigenfunction expansion problem.

Lemma 5. 6. For $f \in C_{0}^{\infty}\left(\mathbb{R}^{n}\right), \lambda>0, \omega \in S^{n-1}=\left\{x \in \mathbb{R}^{n}:|x|=1\right\}$ let

$$
\left(\mathscr{F}_{0}(\lambda) f\right)(\omega)=2^{-1 / 2} \lambda^{(n-2) / 4}(2 \pi)^{-n / 2} \int_{\mathbb{R}^{n}} e^{-i \sqrt{\lambda} \omega x} f(x) d x .
$$

Then we have for $f, g \in C_{0}^{\infty}\left(\mathbb{R}^{n}\right)$,

$$
\left(E_{0}^{\prime}(\lambda) f, g\right)=\left(\mathscr{E}_{0}(\lambda) f, \mathscr{E}_{0}(\lambda) g\right)_{L_{2}(S n-1)} .
$$

Proof. Almost obvious. Recall $\left(\mathscr{F}_{0}(\lambda) f\right)(\omega)=2^{-1 / 2} \lambda^{(n-2) / 4} f(\sqrt{\lambda} \omega)$.

Let $g=f$ in Lemma 5.6. Then by Theorem 3.1 we have

$$
\left\|\mathscr{F}_{0}(\lambda) f\right\|_{L_{2}(S n-1)} \leq C\|f\|_{\left(1+\varepsilon_{0}\right) / 2},
$$

where the constant $C$ is independent of $\lambda$ if $\lambda$ varies over a compact set in $\mathbb{R}_{+}$. (5.1) allows a unique extension $\in \mathbb{B}\left(L_{2,\left(1+\varepsilon_{0}\right) / 2}: L_{2}\left(S^{n-1}\right)\right)$ by continuity of $\mathscr{E}_{0}(\lambda)$, which will be denoted by $\mathscr{E}_{0}(\lambda)$ also. Clearly $\mathscr{F}_{0}(\lambda)$ is strongly continuous in $\lambda>0$. Let $\mathscr{H}=L_{2}\left(\mathbb{R}^{n}\right)$ as above and $\widehat{\mathscr{T}}$ the Hilbert space of all $L_{2}\left(S^{n-1}\right)$-valued square integrable functions over $\mathbb{R}_{+}$ with norm \|\|$\hat{\mathscr{H}}$ and inner product $(,) \hat{\jmath}$.

Theorem 5. 7. (1) For $f, g \in L_{2,\left(1+\varepsilon_{0}\right) / 2}$ we have $\left(E_{0}^{\prime}(\lambda) f, g\right)=\left(\mathscr{E}_{0}(\lambda) f, \mathscr{E}_{0}(\lambda) g\right)_{L_{2}\left(S^{n-1}\right)}, \lambda>0$.

(2) For $f \in L_{2,\left(1+\varepsilon_{0}\right) / 2}$ define $\mathscr{I}_{0} f$ by $\left(\mathscr{F}_{0} f\right)(\lambda)=\mathscr{E}_{0}(\lambda) f$. Then $\mathscr{F}_{0}$ $\in B\left(L_{2,\left(1+\varepsilon_{0}\right) / 2}: \widehat{\mathcal{H}}\right)$. Moreover, $\mathscr{F}_{0}$ can be uniquely extended to a unitary operator from $\mathscr{H}$ onto $\widehat{\mathcal{H}}$, which will be denoted by $\mathscr{I}_{0}$ also.

(3) For $f \in \mathcal{H}$ and any bounded Borel function $\alpha(\lambda)$ defined on the real line we have

$$
\left(\mathscr{I}_{0} \alpha\left(H_{0}\right) f\right)(\lambda)=\alpha(\lambda)\left(\mathscr{I}_{0} f\right)(\lambda) \text { a.e. } \lambda>0 \text {. }
$$

(4) The inversion formula holds for $f \in \mathscr{H}$;

$$
f=\operatorname{s-lim}_{N \rightarrow \infty} \int_{1 / N}^{N} \mathscr{F}_{0}(\lambda) *\left(\mathscr{F}_{0} f\right)(\lambda) d \lambda .
$$


Here $\mathscr{F}_{0}(\lambda)^{*}$ is defined by $\left(\mathscr{E}_{0}(\lambda) * \phi \cdot g\right)=\left(\phi, \mathscr{E}_{0}(\lambda) g\right)$ for any $\phi \in$ $L_{2}\left(S^{n-1}\right)$ and $g \in L_{2,\left(1+\varepsilon_{0}\right) / 2}$.

Proof. (1) is immediate from Lemma 5.6 and Theorem 3.1. The other assertions can be proved by recalling again that $\mathscr{F}_{0}$ is essentially the Fourier transform.

Now we want to arrive at similar results associated with $H(V)$, and begin with

Lemma 5. 8. $\mathscr{I}(\lambda: V) \equiv \mathscr{F}_{0}(\lambda) G(\lambda+i 0: V) \in \boldsymbol{B}\left(L_{2,\left(3-\varepsilon_{0}\right) / 2}: L_{2}\left(S^{n-1}\right)\right)$. $\mathcal{F}(\lambda: V)$ is strongly continuous in $\lambda>0$.

Proof. Direct from Lemma 5.3 and the remark after Lemma 5.6.

With the aid of $\mathscr{I}(\lambda: V)$ we can get a spectral representation for $H(V)$. Our first step is to show the following lemma.

Lemma 5.9. (1) For $f, g \in L_{2,\left(3-\varepsilon_{0}\right) \cdot 2}$ $\left(E^{\prime}(\lambda: V) f, g\right)=(\mathscr{E}(\lambda: V) f, \mathscr{I}(\lambda: V) g)_{L_{2}\left(S^{n-1}\right)}$.

(2) For any Borel set $B \subset(0, \infty)$ and $f, g \in L_{2,\left(3-\varepsilon_{0}\right) / 2}$

$$
(E(B: V) f, g)=\int_{B}(\mathscr{I}(\lambda: V) f, \mathscr{I}(\lambda: V) g)_{L_{2}\left(S^{n-1}\right)} d \lambda .
$$

In particular, for $f \in L_{2,\left(3-\varepsilon_{0}\right) / 2}$ and $B=\boldsymbol{R}_{+}$

$$
\left\|P_{a c} f\right\|^{2}=\int_{0}^{\infty}\|\mathscr{F}(\lambda: V) f\|_{L_{2}\left(S^{n-1}\right)}^{2} d \lambda,
$$

where $P_{a c}=E\left(\boldsymbol{R}_{+}: V\right)$ is the projection onto $\mathcal{H}_{a c}(H(V))$.

Proof. It suffices to show (1), since (2) follows from (1) by integration on $B$. For proving (1) we replace $f, g$ of Theorem 5.7 (1) by $G(\lambda+i 0: V) f$ and $G(\lambda+i 0: V) g$. Then we have with Lemma 5.8 in mind 


$$
\begin{aligned}
\left(E_{0}^{\prime}(\lambda)\right. & G(\lambda+i 0: V) f, G(\lambda+i 0: V) g) \\
& =\left(\mathscr{F}_{0}(\lambda) G(\lambda+i 0: V) f, \mathscr{E}_{0}(\lambda) G(\lambda+i 0: V) g\right)_{L_{2}\left(S^{n-1}\right)} \\
& =(\mathscr{I}(\lambda: V) f, \mathscr{I}(\lambda: V) g)_{L_{2}\left(S^{n-1}\right)}
\end{aligned}
$$

The resolvent equation and Definition 5.2 yield

$$
\begin{aligned}
\frac{1}{2 \pi i}\left(\left[R_{0}(\lambda+i \varepsilon)-R_{0}(\lambda-i \varepsilon)\right] G(\lambda+i \varepsilon: V) f, G(\lambda+i \varepsilon: V) g\right) \\
=\frac{\varepsilon}{\pi}\left(R_{0}(\lambda-i \varepsilon) R_{0}(\lambda+i \varepsilon)\left(H_{0}-(\lambda+i \varepsilon)\right) U(\lambda+i \varepsilon: V) *\right. \\
\quad \times R(\lambda+i \varepsilon: V) f,\left(H_{0}-(\lambda+i \varepsilon)\right) U(\lambda+i \varepsilon: V) * \\
\quad \times R(\lambda+i \varepsilon: V) g) \\
=\frac{\varepsilon}{\pi}(R(\lambda-i \varepsilon: V) R(\lambda+i \varepsilon: V) f, g) \\
=\frac{1}{2 \pi i}([R(\lambda+i \varepsilon: V)-R(\lambda-i \varepsilon: V)] f, g),
\end{aligned}
$$

where we have used the fact that $U(\lambda+i \varepsilon: 1)^{*}$ is unitary. Letting $\varepsilon$ tend to 0 we hare by Lemma 5.3 and Theorem 3.1

$$
\left(E_{0}^{\prime}(\lambda) G(\lambda+i 0: V) f, G(\lambda+i 0: V) g\right)=\left(E^{\prime}(\lambda: V) f, g\right),
$$

so that

$$
\left(E^{\prime}(\lambda: V) f, g\right)=(\mathcal{F}(\lambda: V) f, \mathscr{F}(\lambda: V) g)_{L_{2}\left(S^{n-1}\right)} .
$$

For $f \in L_{2,\left(3-\varepsilon_{0}\right) / 2}$ we set $(\mathcal{F}(V) f)(\lambda)=\mathscr{F}(\lambda: V) f$. Then by Lemma $5.9(2), \mathscr{F}(V) \in \mathbb{R}\left(L_{2,\left(3-\varepsilon_{0}\right) / 2}: \hat{\mathscr{K}}\right)$, and hence is uniquely extended to a partial isometry on $\mathscr{H}$ with initial set $\mathcal{H}_{a c}(H(V))$ and final set contained in $\widehat{\mathcal{H}}$, which we denote by $\mathscr{F}(V)$ also.

Theorem 5. 10 (Spectral Representation (Eigenfunction Expansion) for $H(V))$.

(1) $\mathscr{I}(V)$, defined above, is a partial isometry on $\mathcal{G}$ with initial set $\mathscr{H}_{a c}(H(V))$ and final sel $\widehat{\mathcal{H}}$.

(2) If $\alpha(\lambda)$ is a bounded Borel function defined on the real line we have for $f \in \mathcal{H}_{a c}(H(V))$ 


$$
(\mathscr{E}(V) \alpha(H(V)) f)(\lambda)=\alpha(\lambda)(\mathscr{E}(V) f)(\lambda) \text { a.e. } \lambda>0 .
$$

(3) For $f \in \mathcal{H}_{a c}(H(V))$ the following inversion formula holds:

$$
f=\operatorname{sim}_{N \rightarrow \infty} \int_{1 / N}^{N} \mathscr{E}(\lambda: V) *(\mathscr{F}(V) f)(\lambda) d \lambda .
$$

(4) $\mathcal{E}(\lambda: V) * \in \mathbb{B}\left(L_{2}\left(S^{n-1}\right): L_{2,-\left(3-\varepsilon_{0}\right) / 2}\right)$ is an eigenoperator of $H(V)$ reith eigenvalue $\lambda$ in the sense that for any $\phi \in L_{2}\left(S^{n-1}\right)(-\Delta+V)$ $\times \mathscr{I}(\lambda: V) * \phi=\lambda \mathscr{E}(\lambda: V) * \phi$ holds in the distribution sense.

Proof..$^{1}$ (1) has already been shown above, except for the fact that $\mathscr{I}(V)$ maps onto $\widehat{\mathcal{H}}$, which will be proved in Corollary 5. 12.

To prove (2), it suffices to show the assertion for $\alpha(\lambda)=\chi_{B}(\lambda)$, where $B$ is any Borel set in $\mathbb{R}_{+}$and $\chi_{B}(\lambda)$ is the characteristic function of $B$, since any $\alpha$ can be approximated by a sequence of step functions. First let us note that for $\left.f, g \in \mathcal{H}_{a c}(H(V))(E(B: V) f, g)\right)=\left(\chi_{B} \mathscr{E}(V) f\right.$, $\mathcal{F}(V) g)_{\hat{\varkappa}}$, which follows from Lemma 5.9 (2). We have, therefore,

$$
\begin{aligned}
& \int_{B}\|(\mathscr{F}(V) E(B: V) f)(\lambda)-(\mathscr{F}(V) f)(\lambda)\|_{L_{2}\left(S^{n-1}\right)}^{2} d \lambda \\
& \quad=\int_{B}\|(\mathscr{F}(V)(E(B: V)-1) f)(\lambda)\|_{L_{2}\left(S^{n-1}\right)}^{2} d \lambda \\
& \quad=\|E(B: V)(E(B: V)-1) f\|^{2}=0, \\
& \int_{B^{\prime}}\|(\mathscr{F}(V) E(B: V) f)(\lambda)\|_{L_{2}\left(S^{n-1}\right)}^{2} d \lambda \\
& \quad=\left\|E\left(B^{\prime}: V\right) E(B: V) f\right\|^{2}=0 \quad\left(B^{\prime}=\mathbb{R}_{+}-B\right) .
\end{aligned}
$$

It follows that $(\mathscr{F}(V) E(B: V) f)(\lambda)=(\mathscr{F}(V) f)(\lambda)$ for a.e. $\lambda \in B$ and $(\mathscr{F}(V) E(B: V) f)(\lambda)=0$ for a.e. $\lambda \notin B$, which was to be proved.

To prove (3), let $B$ be a bounded Borel set in $\mathbb{R}_{+}$whose closure does not contain 0 , and consider the operator $\mathscr{F}_{B}(V) *$ defined by $\mathscr{E}_{B}(V) * \widehat{f}=\int_{B} \mathscr{F}(\lambda: V) * \widehat{f}(\lambda) d \lambda$ for $\widehat{f} \in \widehat{\mathcal{H}}$. Obviously $\mathscr{I}_{B}(V) * \epsilon$ $B\left(\widehat{\mathcal{A}}: L_{2,-\left(3-\varepsilon_{0}\right) / 2}\right)$. Let $g \in L_{2,\left(3-\varepsilon_{0}\right) / 2 \text {. Then }}$

$$
\left(\mathscr{F}_{B}(V) * \widehat{f}, g\right)=\int_{B}(\mathscr{F}(\lambda: V) * \widehat{f}(\lambda), g) d \lambda
$$

1) Essentially the same as the proof of Theorem 2.8 of Ikebe [5]. 


$$
\begin{aligned}
& =\int_{B}(\widehat{f}(\lambda), \mathscr{E}(\lambda: V) g)_{L_{2}\left(S^{n-1}\right)} d \lambda \\
& =\int_{B}\left(\widehat{f}(\lambda),(\mathscr{I}(V) g(\lambda))_{L_{2}\left(S^{n-1}\right)} d \lambda\right. \\
& =(\widehat{f}, \mathscr{F}(V) E(B: V) g)_{\hat{\leftrightarrow}} \\
& =\left(\left(\mathscr{I}(V) E\left(B: V^{r}\right)\right)^{*} \hat{f}, g\right),
\end{aligned}
$$

where in passing to the fourth equality we have used $\mathscr{F}(V) E(B: V) g$ $=\chi_{B} \mathscr{E}(V) g$ which has been already proved in (2). Since $L_{2,\left(3-\varepsilon_{0}\right) / 2}$ is dense in $\mathscr{H}$, this shows $\mathscr{F}_{B}(V)^{*}=(\mathscr{F}(V) E(B: V))^{*}$, so that $\mathscr{F}_{B}(V) * \widehat{f}$ $\in \mathcal{H}$. Putting $E_{N}(V)=E\left(\left[N^{-1}, N\right]: V\right)$, we have $\mathscr{I}_{\left[N^{-1}, N\right]}(V)^{*}=$ $\left.\left(\mathscr{F}(V) E_{N}(V)\right)^{*}=E_{N}(V) * \mathscr{E}(V) *=E_{N}(V) \mathscr{E}(V)\right)^{*}$. Letting $N$ tend to $\infty$ we have $\mathscr{E}_{\left[N^{-1}, N\right]}(V)^{*} \rightarrow \mathscr{E}(V)^{*}$, which shows the inversion formula (3).

For (4), as can be checked easily, it suffices to show $\mathscr{E}(\lambda: V)(-\Delta$ $\left.+V^{r}\right) u=\lambda \mathscr{I}(\lambda: V) u$ for $u \in C_{0}^{\infty}\left(\mathbb{R}^{n}\right)$. Set $f=(-\Delta+V-\lambda) u \in C_{0}^{\infty}\left(\mathbb{R}^{n}\right)$. Then by Theorem 3.1 (3) $u=R(\lambda+i 0: V) f$, which means $G(\lambda+i 0: V) f$ $=\left(H_{0}-\lambda\right) e^{i Y} u$ (see Definition 5.2). But since $\mathscr{E}_{0}(\lambda)\left(H_{0}-\lambda\right) v=0$ for an arbitrary $v \in C_{0}^{\infty}\left(\mathbb{R}^{n}\right)$, we have $\mathscr{I}(\lambda: V) f=\mathscr{F}_{0}(\lambda) G(\lambda+i 0: V) f=$ $\mathscr{E}_{0}(\lambda)\left(H_{0}-\lambda\right) e^{i Y} u=0$, i.e. $\mathscr{E}(\lambda: V)(-1+V) u=\lambda \mathscr{F}(\lambda: V) u$. 圈

The above spectral representation alows us to give the following representation of the stationary wave operator $\Gamma\left(H(V): H_{0}\right)$.

Theorem 5。且. Let $\Omega(V)=\mathscr{F}(V) * \mathcal{F}_{0}$. Then $\Gamma\left(H(V): H_{0}\right)$ $=\Omega(V)$.

Proof. Let $f \in L_{2,\left(3-\varepsilon_{0}\right) / 2}, g \in L_{2,\left(1+\varepsilon_{0}\right) / 2}$. Then we have by Theorem 5. 7 (1).

$$
\begin{aligned}
\left(E_{0}^{\prime}(\lambda) G(\lambda+i 0: V) f, g\right) & =\left(\mathscr{F}_{0}(\lambda) G(\lambda+i 0: V) f, \mathscr{I}_{0}(\lambda) g\right)_{L_{2}(S n-1)} \\
& =\left(\mathscr{F}(\lambda: V) f, \mathscr{F}_{0}(\lambda) g\right)_{L_{2}(S n-1)},
\end{aligned}
$$

which integrated over $\mathbb{R}_{+}$yields (see Theorem 5.5 (2), Theorem 5.7 (2), Theorem $5.10(1))\left(\Gamma\left(H_{0}: H(V)\right) f, g\right)=\left(\mathscr{F}(V) f, \mathscr{F}_{0} g\right) \hat{\mathscr{\mu}}$. Since $L_{2,\left(1+\varepsilon_{0}\right) / 2}$ and $L_{2,\left(3-\varepsilon_{0}\right) / 2}$ are dense in $\mathcal{H}$, we have $\Gamma\left(H_{0}: H(V)\right)=\mathscr{F}_{0}^{*} \mathscr{F}(V)$. Taking the adjoint and using Theorem 5.5 (3) we get $\Gamma\left(H(V): H_{0}\right)$ $=\left(\Gamma\left(H_{0}: H(V)\right)\right) *=\mathscr{I}(V) * \mathscr{E}_{0}=\Omega(V)$. 
Corollary 5. 12. The final set of $\mathscr{E}(V)$ is $\widehat{\mathcal{H}}$.

Proof. Since the range of $\mathscr{F}(V)$ is contained in $\widehat{\mathcal{H}}$ and $\mathscr{F}_{0}$ is an isometry with final set $\widehat{\mathcal{H}}$, we have by Theorem $5.11 \mathscr{I}(V)=\mathscr{E}_{0} \Gamma\left(H_{0}\right.$ : $H(V))$. Since $\Gamma\left(H_{0}: H(V)\right)$ has range $\mathscr{H}$ by Theorem 5.5 (2) and since $\mathscr{F}_{0}$ maps $\mathscr{H}$ onto $\widehat{\mathscr{H}}$ by Theorem 5.7 (2), the assertion holds.

We shall further state some results which relate the eigenoperators $\mathscr{F}_{0}(\lambda)^{*}, \mathscr{I}(\lambda: V)^{*}$ to the asymptotic properties of the solutions of the inhomogeneous Schrödinger equations, which will be utilized in $\S 7$.

Let us define the operator $\mathcal{F}_{0}(\lambda, r)$ by

$$
\begin{aligned}
& \left(\mathscr{I}_{0}(\lambda, r) f\right)(\omega)=C(\lambda) r^{(n-1) / 2} e^{-i \sqrt{\lambda} r}\left(R_{0}(\lambda+i 0) f\right)(r \omega), \\
& C(\lambda)=e^{(n-3) \pi i / 4} \pi^{-1 / 2} \lambda^{1 / 4}, \quad\left(\lambda>0, f \in L_{2,\left(1+\varepsilon_{0}\right) / 2}\right) .
\end{aligned}
$$

Lemma 5. 13. Let $f \in C_{0}^{\infty}\left(\mathbb{R}^{n}\right)$. Then the following strong limit exists in $L_{2}\left(S^{n-1}\right)$ :

$$
\operatorname{s}_{r \rightarrow \infty} \lim _{0}(\lambda, r) \int=\mathscr{E}_{0}(\lambda) f .
$$

Proof. It is well-known that the operator $R_{0}(\lambda+i 0)$ has an integral kernel

$$
G(x-y, \sqrt{\lambda})=\frac{i}{4}\left(\frac{\sqrt{\lambda}}{2 \pi|x-y|}\right)^{n / 2-1} H_{n / 2-1}^{(1)}(\sqrt{\lambda}|x-y|),
$$

where $H_{\nu}^{(1)}(z)$ is the Hankel function of the first kind (see Titchmarsh [12], p. 79). The asymptotic form of $H_{\nu}^{(1)}(z)$ as $z \rightarrow \infty$ is given by

$$
H_{\nu}^{(1)}(z)=2^{1 / 2}(\pi z)^{-1 / 2} \exp \{i(z-(2 \nu+1) \pi / 4)\}\left(1+O\left(z^{-1}\right)\right)
$$

(see Watson [13], p. 196-198). Noting that $f \in C_{0}^{\infty}\left(\boldsymbol{R}^{n}\right)$ we have thus by a straightforward calculation

$$
\mathscr{F}_{0}(\lambda, r) f=\mathscr{F}_{0}(\lambda) f+O\left(r^{-1}\right) .
$$

We have already known that both $\mathscr{F}_{0}(\lambda) f$ and $\mathscr{F}_{0}(\lambda, r) f$ have meaning when $f \in L_{2,\left(1+\varepsilon_{0}\right) / 2}$. It may thus be inferred by Lemma 5.13 that even for $f \in L_{2,\left(1+\varepsilon_{0}\right) / 2}, \mathscr{F}_{0}(\lambda, r) f \rightarrow \mathscr{E}_{0}(\lambda) f$ as $r \rightarrow \infty$. This will turn out to be the case in a sense by the following two propositions. 
Proposition 5. 14. Let $f \in L_{2,1}$.

(1) There exists a sequence $\left\{r_{m}\right\}$ tending to $\infty$ such that for $m \rightarrow \infty$

$$
\begin{aligned}
& r_{m}^{-\varepsilon_{0}} \int_{|x|=r_{m}}\left|R_{0}(\lambda+i 0) f\right|^{2} d S \rightarrow 0, \\
& r_{m} \int_{|x|=r_{m}}\left|\mathscr{D}(\lambda+i 0) R_{0}(\lambda+i 0) f\right|^{2} d S \rightarrow 0 .
\end{aligned}
$$

(2) There exists the following strong limit in $L_{2}\left(S^{n-1}\right)$ :

$$
\mathrm{s}-\lim _{m \rightarrow \infty} \mathscr{I}_{0}\left(\lambda, r_{m}\right) f \equiv \widetilde{\mathscr{I}}_{0}(\lambda) f,
$$

where $\left\{r_{m}\right\}$ is any sequence specified in (1). The limit $\widetilde{\mathscr{I}}_{0}(\lambda) f$ is independent of the choice of $\left\{r_{m}\right\}$.

$$
\left\|\mathscr{I}_{0}(\lambda) f\right\|_{L_{2}(S n-1)} \leq C\|f\|_{\left(1+\varepsilon_{0}\right) / 2} .
$$

In particular $\widetilde{\Psi}_{0}(\lambda)$ is extended by continuity to an operator $\in$ $\mathbb{B}\left(L_{2,\left(1+\varepsilon_{0}\right) / 2}: L_{2}\left(S^{n-1}\right)\right)$.

Proof. The proof is given in Lemmas 1.3,2.2 and 2.7 of Ikebe [5] under a more general situation.

Proposition 5. 15. For $f \subseteq L_{2,\left(1+\varepsilon_{0}\right) / 2}$ the following assertions hold.

(1) There exists a sequence $\left\{r_{m}\right\}$ tending to $\infty$ such that for $m \rightarrow \infty$

$$
\begin{aligned}
& r_{m}^{-\varepsilon_{0}} \int_{|x|=r_{m}}\left|R_{0}(\lambda+i 0) f\right|^{2} d S \rightarrow 0, \\
& r_{m}^{\varepsilon_{0}} \int_{|x|=r_{m}}\left|\mathscr{D}(\lambda+i 0) R_{0}(\lambda+i 0) f\right|^{2} d S \rightarrow 0 .
\end{aligned}
$$

(2) For an arbitrary $\phi \in L_{2}\left(S^{n-1}\right)$

$$
\left(\phi, \widetilde{\mathscr{F}}_{0}(\lambda) f\right)_{L_{2}\left(s^{n-1}\right)}=\lim _{m \rightarrow \infty}\left(\phi, \mathscr{I}_{0}\left(\lambda, r_{m}\right) f\right)_{L_{2}(s n-1)}
$$

holds, where $\left\{r_{m}\right\}$ is any sequence specified in (1).

Proof. For the proof see Lemma 3.2 of Ikebe [5].

Now for $f \in L_{2,\left(3-\varepsilon_{0}\right) / 2}$ we define $\mathscr{I}(\lambda, r: V) f$ by

$$
(\mathscr{I}(\lambda, r: V) f)(\omega)=C(\lambda) r^{(n-1) / 2} e^{i K(r \omega, \lambda: \eta)}(R(\lambda+i 0: V) f)(r \omega),
$$


where $C(\lambda)=e^{(n-3) \pi i / 4} \pi^{-1 / 2} \lambda^{1 / 4}$,

$$
K(x, \lambda: V)=-\sqrt{\lambda} r+Y(x, \lambda: V), \quad r=|x| \geq 2 .
$$

Lemma 5. 16. Let $f \subseteq L_{2,\left(3-\varepsilon_{0}\right) / 2}$, and set $u(\lambda+i 0: V: f)=R_{0}(\lambda$ $+i 0) G(\lambda+i 0: V) f$. Then:

(1) There exists a sequence $\left\{r_{m}\right\}$ tending to $\infty$ such that as $m \rightarrow \infty$

$$
\begin{aligned}
& r_{m}^{-\varepsilon_{0}} \int_{|x|=r_{m}}|u(\lambda+i 0: V: f)|^{2} d S \rightarrow 0, \\
& \left.r_{m}^{\varepsilon_{0}} \int_{|x|=r_{m}} \mathscr{D}(\lambda+i 0) u(\lambda+i 0: V: f)\right|^{2} d S \rightarrow 0 .
\end{aligned}
$$

(2) For any $\phi \in L_{2}\left(S^{n-1}\right)$

$$
(\phi, \mathscr{E}(\lambda: V) f)_{L_{2}\left(S^{n-1}\right)}=\lim _{m \rightarrow \infty}\left(\phi, \mathscr{E}\left(\lambda, r_{m}: V\right) f\right)_{L_{2}\left(S^{n-1}\right)},
$$

where $\left\{r_{m}\right\}$ is any sequence specified in (1).

Proof. Let us replace $f$ of Proposition 5.15 by $G(\lambda+i 0: V) f$. Then (1) follows from Proposition 5.15 (1), since $G(\lambda+i 0: V) f \in L_{2,\left(1+\varepsilon_{0}\right) / 2}$ by Lemma 5. 3. By definition we have $R_{0}(\lambda+i 0) G(\lambda+i 0: V) f=e^{i Y} R(\lambda+i 0$ : $V) f$, and hence $\mathscr{F}_{0}(\lambda, r) G(\lambda+i 0: V) f=\mathscr{I}(\lambda, r: V) f$. Thus by Proposi tion 5.15 (2)

$$
\begin{aligned}
\lim _{m \rightarrow \infty}(\phi, \mathscr{F} & \left.\left(\lambda, r_{m}: V\right) f\right)_{L_{2}\left(S^{n-1}\right)} \\
& =\left(\phi, \widetilde{\mathscr{H}}_{0}(\lambda) G(\lambda+i 0: V) f\right)_{L_{2}\left(S^{n-1}\right)} .
\end{aligned}
$$

Therefore, it suffices to show $\mathscr{F}_{0}(\lambda)=\widetilde{\mathscr{F}}_{0}(\lambda)$ because of Lemma 5.8. However the latter is obvious from the fact that $\widetilde{\mathscr{F}}_{0}(\lambda) f=\mathscr{F}_{0}(\lambda) f$ for $f \in C_{0}^{\infty}\left(\mathbb{R}^{n}\right)$ by Lemma 5.13 and Proposition 5.14 (3).

The above lemma shows that $\mathscr{I}\left(\lambda, r_{m}: V\right) f$ converges weakly to $\mathscr{F}(\lambda: V) f$ in $L_{2}\left(S^{n-1}\right)$, but we can further prove its strong convergence. In fact Ikebe [5] and Saitō [11] have established the strong convergence of $\mathscr{I}\left(\lambda, r_{m}: V\right) f$. Their definition of $\mathscr{I}(\lambda: V) f$ is by $\operatorname{s-lim}_{m \rightarrow \infty} \mathscr{E}\left(\lambda, r_{m}: V\right) f$, which is apparently different from ours (Lemma 5. 8). By what we have stated, however, our eigenoperators $\mathscr{E}(\lambda: V)$ and theirs coincide. 


\section{$\S 6$. Continuous Dependence of $V$ on the Stationary Wave Operator}

In the following arguments $D$ denotes an arbitrary bounded domain of $\mathbb{C}-\mathbb{R}$ such that $\bar{D}$ does not intersect with $(-\infty, 0]$. We set $(v(z: f: V))(x)=e^{i Y\left(x,(\operatorname{Re} \sqrt{z})^{3}: V\right)}(R(z: V) f)(x)$ for brevity.

Lemma 6. 1. Let $f \in L_{2,\left(3-\varepsilon_{0}\right) / 2}$, and let $V$ vary on a compact set $K$ in $\mathbb{V}$. Then there exists a constant $C$ independent of $z \in \bar{D}$ and $V \in K$ such that

$$
\|\mathscr{D}(z) v(z: f: V)\|_{\left(1-\varepsilon_{0}\right) / 2, E_{1}} \leq C\|f\|_{\left(3-\varepsilon_{0}\right) / 2} .
$$

Furthermore, the following strong limit exists in $L_{2,\left(1-3 \varepsilon_{0}\right) / 2}\left(E_{1}\right)$ :

$$
\operatorname{s-lim}_{z \rightarrow \lambda+i 0} \mathscr{D}(z) v(z: f: V)=\mathscr{D}(\lambda+i 0) v(\lambda+i 0: f: V), \quad \lambda>0 .
$$

Proof. We have only to show that $C$ is independent of $V \in K$, since the remaining assertions of the lemma are given in Lemma 2.7 of Isozaki [7]. In Proposition 6 of [7] it has been proved that

$$
\begin{aligned}
\| \mathscr{D}(z) & v(z: f: V) \|_{\left(1-\varepsilon_{0}\right) / 2, E_{1}} \\
& \leq C\left(\|R(\approx: V)\|_{-\left(1-\varepsilon_{0}\right) / 2}+\|f\|_{\left(3-\varepsilon_{0}\right) / 2}\right),
\end{aligned}
$$

where the constant $C$ is independent of $z \in \bar{D}$. Although Isozaki [7] has not explicitly stated the fact that $C$ can be taken independently of $V$ in a bounded set of $\boldsymbol{V}$, this can be seen by carefully examining the argument given in [7]. On the other hand we have seen in Lemma 3.2

$$
\|R(z: V) f\|_{-\left(1-\varepsilon_{0}\right) / 2} \leq C\|f\|_{\left(3-\varepsilon_{0}\right) / 2},
$$

where the constant $C$ is independent of $z \in \bar{D}$ and $V$ if $V$ is in a compact set in $\mathbb{V}$. The above two facts prove the independence on $V$ of $C$. 图

ILemma 6.2. Let $f \in L_{2,\left(3-\varepsilon_{0}\right) / 2}$. Then for $\rho \geq 1$

$$
\|\mathscr{D}(\lambda+i 0) v(\lambda+i 0: f: V)\|_{\left(1-3 \varepsilon_{0}\right) / 2, E_{p}} \leq C \rho^{-\varepsilon_{0}}\|f\|_{\left(3-\varepsilon_{0}\right) / 2},
$$

where the constant $C$ is independent of $V$ if it varies over a compact set in $\mathbb{V}$.

Proof. This fact is proved from Lemma 6.1 as follows: 


$$
\begin{aligned}
\|\mathscr{D}(\lambda+i 0) v(\lambda+i 0: f: V)\|_{\left(1-3 \varepsilon_{0}\right) / 2, \boldsymbol{E}_{\rho}}^{2} \\
\quad=\int_{|x|>\rho}(1+|x|)^{1-\varepsilon_{0}-2 \varepsilon_{0}}|\mathscr{D}(\lambda+i 0) v(\lambda+i 0: f: V)|^{2} d x \\
\quad \leq \rho^{-2 \varepsilon_{0}} \int_{|x|>\rho}(1+|x|)^{1-\varepsilon_{0}}|\mathscr{D}(\lambda+i 0) v(\lambda+i 0: f: V)|^{2} d x \\
\quad \leq C \rho^{-2 \varepsilon_{0}}\|f\|_{\left(3-\varepsilon_{0}\right) / 2}^{2} .
\end{aligned}
$$

Lemma 6. 3. Let $f \in L_{2,\left(3-\varepsilon_{0}\right) / 2}$. Let $V_{m} \rightarrow V$ in $\mathbb{V}$. Then we have $\mathscr{D}(\lambda+i 0) v\left(\lambda+i 0: f: V_{m}\right) \rightarrow \mathscr{D}(\lambda+i 0) v(\lambda+i 0: f: V)$ in $L_{2,\left(1-3 \varepsilon_{0}\right) / 2}\left(E_{1}\right)$ as $m \rightarrow \infty$.

Proof. By Lemma 6.2, for an arbitrary $\varepsilon>0$, there exists a constant $\rho>1$ such that for all $m=1,2, \cdots\left\|\mathscr{D}(\lambda+i 0) v\left(\lambda+i 0: f: V_{m}\right)\right\|_{\left(1-3 \varepsilon_{0}\right) / 2, E_{\rho}}$ $<\varepsilon$. We also have by Theorem 3.3 and the elliptic estimate (3.8) $R\left(\lambda+i 0: V_{m}\right) f \rightarrow R(\lambda+i 0: V) f$ in $H_{\text {loc }}^{2}$, so that it follows for $m \rightarrow \infty$ $\mathscr{D}(\lambda+i 0) v\left(\lambda+i 0: f: V_{m}\right) \rightarrow \mathscr{D}(\lambda+i 0) v(\lambda+i 0: f: V)$ in $L_{2}\left(B_{1, \rho}\right)$. These facts prove the lemma. 圈

Lemma 6. 4. Let $f \in L_{2,\left(8-\varepsilon_{0}\right) / 2}, \quad V_{m} \rightarrow V$ in $\boldsymbol{V}$. Then for $\lambda \in$ $\overline{D \cap \boldsymbol{R}_{+}},\left\|G\left(\lambda+i 0: V_{m}\right) f\right\|_{\left(1+\varepsilon_{0}\right) / 2} \leq C\|f\|_{\left(3-\varepsilon_{0}\right) / 2}$, where the constant $C$ is independent of $\lambda \in \overline{D \cap \mathbb{R}_{+}}$and $m$, and also we have $G\left(\lambda+i 0: V_{m}\right) \rightarrow$ $G(\lambda+i 0: V) f$ in $L_{2,\left(1+\varepsilon_{0}\right) / 2}$.

Proof. By a direct calculation it follows that

$$
\begin{aligned}
G\left(\lambda+i 0: V_{m}\right) f= & e^{i Y} f-i\left(\frac{\partial^{2} Y}{\partial r^{2}}+\frac{\Lambda Y}{r^{2}}\right) v\left(\lambda+i 0: f: V_{m}\right)^{1)} \\
& -\left(|\nabla Y|^{2}+V_{m}-2 \sqrt{\lambda} \frac{\partial Y}{\partial r}\right) v\left(\lambda+i 0: f: V_{m}\right) \\
& -2 i \nabla_{x} Y \cdot \mathscr{D}(\lambda+i 0) v\left(\lambda+i 0: f: V_{m}\right),
\end{aligned}
$$

where $Y=Y\left(x, \lambda: V_{m}\right) \quad$ (see (3.3) of [7]). We have by Lemma 4.7

$$
2 \sqrt{\lambda} \frac{\partial Y}{\partial r}-V_{m}-\left|\nabla_{x} Y\right|^{2} \mid \leq C(1+|x|)^{-2},
$$

\footnotetext{
1) $A$ is the Laplace-Beltrami operator on $S^{n-1}$.
} 


$$
\begin{aligned}
& \left|\frac{\partial^{2} Y}{\partial r^{2}}+\frac{\Lambda Y}{r^{2}}\right| \leq C(1+|x|)^{-1-\hat{o}}, \\
& \left|\Gamma_{x} Y\right| \leq C(1+|x|)^{-\hat{\delta}} \\
& Y=0 \text { if }|x| \leq 1
\end{aligned}
$$

where the constant $C$ is independent of $m$. We have thus by Lemmas 3.2 (1) and 6.1 proved the irst half of the lemma. Lemma 4.7 also shows $D_{x}^{\alpha} Y\left(x, \lambda: V_{m}\right) \rightarrow D_{x}^{\alpha} Y(x, \lambda: V)$ as $m \rightarrow \infty$. In view of these facts and taking note of Theorem 3.3 and Lemma 6.3 we see that each term of the right hand side of $(f, 1)$ converges to the corresponding term of $G(\lambda+i 0: T) . f$ in $L_{\varepsilon .\left(1+\varepsilon_{0}\right), 2}$. This proves the last half. 橉

Lemuna 6. 5. Let $f \in L_{\left.\text {: (3- }-c_{0}\right) / 2 .}$ Let $T_{m}^{\top} \rightarrow T^{\top}$ in $\mathbb{V}$. Then there exists a constant $C$ independent of $m$ and $\lambda \equiv \bar{D} \cap \bar{R}_{+}$such that

$$
\left\|\mathscr{I}\left(\lambda: V_{m}\right) f\right\|_{L_{2}\left(S^{n-1}\right)} \leq C\|f\|_{\left(3-\varepsilon_{0}\right) / 2} .
$$

. Toreoz'cr, we have for $\lambda>0$ F $\left(\lambda: V_{m}\right) f \rightarrow \mathscr{E}\left(\lambda: V^{\top}\right)$ f in $L_{2}\left(S^{n-1}\right)$.

Proof. The lemma follows from Lemmas 5.8 and 6.4. 四

Thoorem 6. 6. Let $V_{m} \rightarrow T^{\top}$ in $\mathbb{V}$. Then $\Omega\left(V_{m}\right) \rightarrow \Omega(V)$ strongly in $\mathscr{H}=L_{2}\left(\mathbb{R}^{n}\right)$.

Proof. Since $\Omega\left(V_{m}\right), \Omega(V)$ are isometries, it is sufficient to show $\left(\Omega\left(V_{m}\right) f, g\right) \rightarrow\left(\Omega\left(T^{\top}\right) f, g\right)$ for $f, g$ in a dense set of $L_{2}\left(\mathbb{R}^{n}\right)$. Let $f \in$ $\mathscr{F}^{-1}\left(C_{0}^{\infty}\left(\mathbb{R}^{n}-\{0\}\right)\right), g \in L_{2,\left(3-\varepsilon_{0}\right) / 2}$. Then by the definition of $\Omega(V)$ in Theorem 5.11 and the definition of $\mathscr{E}(V)$ before Theorem 5.10 we have

$$
\begin{aligned}
\left(\Omega\left(V_{m}\right) f, g\right) & =\left(\mathscr{E}\left(I_{m}\right) * \mathscr{E}_{0} f, g\right) \\
& =\left(\mathscr{E}_{0} f, \mathscr{F}\left(V_{m}\right) g\right) \\
& =\int_{0}^{\infty}\left(\mathscr{F}_{0}(\lambda) f, \mathscr{I}\left(\lambda: V_{m}^{r_{m}}\right) g\right)_{I_{2}\left(S^{n-1}\right)} d \lambda .
\end{aligned}
$$

Since $f \in \mathscr{I}^{-1}\left(C_{0}^{\infty}\left(\mathbb{R}^{n}-\{0\}\right)\right)$, the integration is actually performed on a compact set of $\mathbb{R}$.. With this in mind we see by Lemma 6.5 and Lebesgue's dominated convergence theorem that $\left(\Omega\left(T_{m}{ }_{m}\right) f, g\right) \rightarrow\left(\Omega\left(T^{\top}\right) f, g\right)$. 


\section{§ 7. $W(\mathbb{V})=\Omega(\mathbb{V})$ for Compactly Supported $\mathbb{V}$}

When $V \in \mathbb{V}$ has a compact support, the scattering problems for $H(V)$ have been thoroughly investigated so far. We shall briefly look at known results and prepare the final stage for proving the completeness of $W(V)$.

Theorem 7. 1. Let $V \in \mathbb{V}$ be compactly supported. Then the following strong limit exists in $\mathcal{H}$ :

$$
\text { s- } \lim _{t \rightarrow \infty} e^{i t H(n)} e^{-i t H_{0}} \equiv W^{(s)}(V) .
$$

$W^{(s)}(V)$ is an isometry on $\mathcal{H}$ with final set $\mathcal{H}_{a c}(H(V))$.

For the proof see e.g., Kato-Kuroda [8].

Definition 7.2. Let $G^{(s)}(z: V), \widetilde{G}^{(s)}(z: V)$ be defined by

$$
\begin{aligned}
& G^{(s)}(z: V)=\left(H_{0}-z\right) R(z: V), \\
& \widetilde{G}^{(s)}(z: V)=(H(V)-z) R_{0}(z), \quad z \in C-\mathbb{R}
\end{aligned}
$$

Lemma 7. 3. Let $V \in \mathbb{V}$ have a compact support. Then the following strong limits exist in $\boldsymbol{B}\left(L_{2,\left(1+\varepsilon_{0}\right) / 2}: L_{2,\left(1+\varepsilon_{0}\right) / 2}\right)$ for $\lambda>0$ :

$$
\begin{aligned}
& s-\lim _{\varepsilon \downarrow 0} G^{(s)}(\lambda+i \varepsilon: V) \equiv G^{(s)}(\lambda+i 0: V), \\
& s-\lim _{\varepsilon \downarrow 0} \widetilde{G}^{(s)}(\lambda+i \varepsilon: V) \equiv \widetilde{G}^{(s)}(\lambda+i 0: V) .
\end{aligned}
$$

$G^{(s)}(\lambda+i 0: V)$ and $\widetilde{G}^{(s)}(\lambda+i 0: V)$ are strongly continuous in $\lambda>0$.

Proof. By the resolvent equation we have $G^{(s)}(z: V)=1-V R(z$ : $V), \widetilde{G}^{(s)}(z: V)=1+V R_{0}(z)$. Hence the assertion of the lemma readily follows from Theorem 3.1 if it is noted that $V R(z: V)$ and $V R_{0}(z)$ lie in $\boldsymbol{B}\left(L_{2,\left(1+\varepsilon_{0}\right) / 2}: L_{2,\left(1+\varepsilon_{0}\right) / 2}\right)$.

We define for $f \in L_{2,\left(1+\varepsilon_{0}\right) / 2}, 0<a<b<\infty$

$$
\Gamma^{(s)}\left((a, b): H(V): H_{0}\right) f=\int_{a}^{b} E^{\prime}(\lambda: V) \widetilde{G}^{(s)}(\lambda+i 0: V) f d \lambda .
$$


Then $\operatorname{sim} \Gamma^{(s)}\left((a, b): H(V): H_{0}\right) f \equiv \Gamma^{(s)}\left(H(V): H_{0}\right) f$ is seen to exist (see $\underset{\substack{b \rightarrow \infty \\ a \rightarrow 0}}{h \rightarrow 0}$

e.g. Theorem 6 of [7]), and $\Gamma^{(s)}\left(H(V): H_{0}\right)$ is uniquely extended to an isometry on $\mathcal{H}$ with final set $\mathcal{K}_{a c}(H(V))$.

Let us define $\mathscr{F}^{(s)}(\lambda: V) \in \mathbb{B}\left(L_{2,\left(1+\varepsilon_{0}\right) / 2}: L_{2}\left(S^{n-1}\right)\right)$ by

$$
\mathscr{F}^{(s)}(\lambda: V)=\mathscr{E}_{0}(\lambda) G^{(s)}(\lambda+i 0: V)
$$

and $\mathscr{I}^{(s)}(V)$ by

$$
\left(\mathcal{F}^{(s)}(V) f\right)(\lambda)=\mathscr{F}^{(s)}(\lambda: V) f \quad\left(f \in L_{2,\left(1-\varepsilon_{0}\right) / 2}\right) .
$$

The arguments given in Section 5 also hold for $\mathscr{F}^{(s)}(V)$. We can thus prove the following theorem in the same way as Theorem 5.11.

Theorem 7.4. Let $V \in \mathbb{V}$ be compactly supported. Define $\Omega^{(s)}(V)$ by $\Omega^{(s)}(V)=\mathscr{F}^{(s)}(V) * \mathscr{F}_{0}$. Then we have $\Omega^{(s)}(V)=\Gamma^{(s)}\left(H\left(V^{\top}\right)\right.$ : $\left.H_{0}\right)$.

Theorem 7.5 . If $V \in \mathbb{V}$ has a compact support, we have $W^{(s)}(V)$ $=\Omega^{(s)}(V)$.

This is well-known (see e.g. Kato-Kuroda [8]).

Next we shall consider the relation between modified and non-modified wave operators.

Lemma 7.6. Let $V \in \mathbb{V}$ be compactly supported. Let $X(\xi, t: V)$ be the time-dependent modifier constructed in Section 2. Then the limit $\lim X(\xi, t: V) \equiv X_{\infty}(\xi: V)$ exists if $\xi \neq 0$, and the convergence is uniform for $\xi$ when $\xi$ lies in a compact sel in $\mathbb{R}^{n}-\{0\}$.

Proof. Set $A(\xi, t: V)=\frac{\partial}{\partial t} X(\xi, t: V)-V\left(2 \xi t+\nabla_{\xi} X(\xi, t: V)\right)$. Then we have

$$
\begin{aligned}
X(\xi, t: V)=X(\xi, 1: V)+\int_{1}^{t}[V(2 \xi s & \left.+\nabla_{\xi} X(\xi, s: V)\right) \\
& +A(\xi, s: V)] d s
\end{aligned}
$$

Since $T^{T}$ has a compact support, taking note of Lemma 2.2, we see the 
integrand of the right hand side integrable if $\xi \neq 0$. Hence the limit $\lim _{t \rightarrow \infty} X(\xi, t: V)$ exists if $\xi \neq 0$. The uniformity of convergence is guaranteed by Lemma 2.2 (2).

Lemma 7.7. Let $V \in \mathbb{V}$ be compactly supported, and let $Y(x, \lambda$ : $V)$ be the stationary modifier constructed in $\S 4$. Then for $\lambda>0$ the limit $\lim _{r \rightarrow \infty} Y(r \omega, \lambda: V) \equiv Y_{\infty}(\omega, \lambda: V)\left(\omega \in S^{n-1}\right)$ exists, and $Y_{\infty}(\omega, \lambda: V)$ $=X_{\infty}(\sqrt{\lambda} \omega: V)$.

Proof. Let $\xi(x, \lambda: V)$ and $t(x, \lambda: V)$ be the functions specified in Lemma 4.2. We set $\phi(x, \lambda: V)=t(x, \lambda: V)-r /(2 \sqrt{\lambda}), \psi(x, \lambda: V)=$ $\xi(x, \lambda: V)-\sqrt{\lambda} \omega, r=|x|, \omega=x / r$. By Lemma 4.3 we have for $r \rightarrow \infty$ $\phi(x, \lambda: V)=O(1), \psi(x, \lambda: V)=O\left(r^{-1}\right)$. By Definition 4.6 Y $(x, \lambda: V)$ takes the form

$$
\begin{aligned}
Y(x, \lambda: V)= & X(\sqrt{\lambda} \omega+\psi, r /(2 \sqrt{\lambda})+\phi: V)+r /(2 \sqrt{\lambda})|\psi|^{2} \\
& +2 \sqrt{\lambda} \omega \psi \phi+\phi|\psi|^{2}
\end{aligned}
$$

for sufficiently large $r$. Letting $r$ tend to infinity, and taking note of Lemma 7.6 and the above asymptotic estimate, we have $\lim _{r \rightarrow \infty} Y(r \omega, \lambda: V)$ $=X_{\infty}(\sqrt{\lambda} \omega: V)$.

Lemma 7.8. Let $V \in V$ be compactly supported. Then we have for $\lambda>0 \mathscr{F}(\lambda: V)=e^{i Y_{\infty}(\cdot, \lambda \cdot V)} \mathscr{F}^{(s)}(\lambda: V)$ in $\boldsymbol{B}\left(L_{2,\left(1+\varepsilon_{0}\right) / 2}: L_{2}\left(S^{n-1}\right)\right)$.

Proof. Since both sides are in $\mathbb{B}\left(L_{2,\left(1+\varepsilon_{0}\right) / 2}: L_{2}\left(S^{n-1}\right)\right.$ ) (see Lemma 5. $9^{1)}$ and the statements preceeding theorem 7.4 ), we have only to prove

$$
(\mathscr{E}(\lambda: V) f)(\omega)=e^{i Y_{\infty}(\omega, \lambda: V)}\left(\mathscr{F}^{(s)}(\lambda: V) f\right)(\omega), \omega \in S^{n-1},
$$

when $f \in L_{2,\left(3-\varepsilon_{0}\right) / 2}$ which is dense in $L_{2,\left(1+\varepsilon_{0}\right) / 2}$. Let $f \in L_{2,\left(3-\varepsilon_{0}\right) / 2}$. Put

$$
\begin{aligned}
& u=u(\lambda+i 0: V: f)=R_{0}(\lambda+i 0) G(\lambda+i 0: V) f, \\
& u^{(s)}=u^{(s)}(\lambda+i 0: V: f)=R_{0}(\lambda+i 0) G^{(s)}(\lambda+i 0: V) f, \\
& \mathcal{F}(\lambda, r: V) f=C(\lambda) r^{(n-1) / 2} e^{-i \sqrt{\lambda} r^{\perp} i Y(r \cdot, \lambda: V)}(R(\lambda+i 0: V) f)(r \cdot),
\end{aligned}
$$

1) Plus some arguments involving the limiting absorption method (Theorem 3.1). 


$$
\begin{aligned}
& \mathscr{E}^{(s)}(\lambda, r: V) f=C(\lambda) r^{(n-1) / 2} e^{-i \sqrt{\lambda} r}(R(\lambda+i 0: V) f)(r \cdot), \\
& C(\lambda)=e^{(n-3) \pi i / 4} \pi^{-1 / 2} \lambda^{1 / 4} .
\end{aligned}
$$

Since $G(\lambda+i 0: V) f \in L_{2,\left(1+\varepsilon_{0}\right) / 2}$ by Lemma 5.3 and $G^{(s)}(\lambda+i 0: V) f$ $\in L_{2,\left(1+\varepsilon_{0}\right) / 2}$ by Lemma 7.3 , we have $u, u^{(s)} \in L_{2,-\left(1+\varepsilon_{0}\right) / 2} \mathscr{D}(\lambda+i 0) u$, $\mathscr{D}(\lambda$ $+i 0) u^{(s)} \in L_{2,-\left(1-\varepsilon_{0}\right) / 2}\left(E_{1}\right)$ by Theorem 3. 1. In consequence,

$$
\begin{aligned}
\int_{|x|>1} & {\left[(1+r)^{-\left(1+\varepsilon_{0}\right)}\left\{|u|^{2}+\left|u^{(s)}\right|^{2}\right\}\right.} \\
& \left.+(1+r)^{-\left(1-\varepsilon_{0}\right)}\left\{|\mathscr{D} u|^{2}+\left|\mathscr{D} u^{(s)}\right|^{2}\right\}\right] d x<\infty,
\end{aligned}
$$

from which we see that there exists a diverging sequence $\left\{r_{m}\right\}$ such that as $m \rightarrow \infty$

$$
\begin{array}{ll}
r_{m}^{-\varepsilon_{0}} \int_{|x|=r_{m}}|u|^{2} d S \rightarrow 0, & r_{m}^{-\varepsilon_{0}} \int_{|x|=r_{m}}\left|u^{(s)}\right|^{2} d S \rightarrow 0, \\
r_{m}^{\varepsilon_{0}} \int_{|x|=r_{m}}|\mathscr{D} u|^{2} d S \rightarrow 0, & r_{m}^{\varepsilon_{0}} \int_{\mid x_{i}=r_{m}}\left|\mathscr{D} u^{(s)}\right|^{2} d S \rightarrow 0 .
\end{array}
$$

Now Lemma 5.16 (2) furnishes

$$
\begin{aligned}
& (\phi, \mathscr{F}(\lambda: V) f)_{L_{2}\left(S^{n-1}\right)}=\lim _{m \rightarrow \infty}\left(\phi, \mathscr{F}\left(\lambda, r_{m}: V\right) f\right)_{L_{2}\left(S^{n-1}\right)}, \\
& \left(\phi, \mathscr{E}^{(s)}(\lambda: V) f\right)_{L_{2}\left(S^{n-1}\right)}=\lim _{m \rightarrow \infty}\left(\phi, \mathscr{F}^{(s)}\left(\lambda, r_{m}: V\right) f\right)_{L_{2}\left(S^{n-1}\right)}
\end{aligned}
$$

for all $\phi \in L_{2}\left(S^{n-1}\right)$. Since $\lim _{r \rightarrow \infty} Y(r \omega, \lambda: V)=Y_{\infty}(\omega, \lambda: V)$ by Lemma 7.7 , we see that

$$
\begin{aligned}
\lim _{m \rightarrow \infty}(\phi, & \left.\mathscr{E}\left(\lambda, r_{m}: V\right) f\right)_{L_{2}\left(S^{n-1}\right)} \\
& =\lim _{m \rightarrow \infty}\left(\phi, e^{\left.i Y^{\left(r_{m}\right.}, \lambda: V\right)} \mathscr{E}^{(s)}\left(\lambda, r_{m}: V^{r}\right) f\right)_{L_{2}\left(S^{n-1}\right)} \\
& =\left(\phi, e^{i Y_{00}(\cdot, \lambda: V)} \mathscr{E}^{(s)}(\lambda: V) f\right)_{L_{2}\left(S^{n-1}\right)},
\end{aligned}
$$

which shows $\mathscr{E}(\lambda: V) f=e^{i Y_{\infty}(\cdot, \lambda: V)} \mathscr{F}^{(s)}(\lambda: V) f$. 圈

Theorem 7. 9. Let $V \in V$ be compactly supported. Then we have $W(V)=\Omega(V)$.

Proof. Since both $W(V)$ and $\Omega(V)$ are isometric by Theorems 2. 3, 5.5 and 5.11 , we have only to prove $(W(V) f, g)=(\Omega(V) f, g)$ for $f, g$ 
in a dense set of $\mathscr{H}$. Let $f \in \mathscr{I}^{-1}\left(C_{0}^{\infty}\left(\boldsymbol{R}^{n}-\{0\}\right)\right), g \in L_{2,\left(1+\varepsilon_{0}\right) / 2}$. We have by Theorem 7.1 and Lemma 7.6

$$
\begin{aligned}
(W(V) f, g) & =\lim _{t \rightarrow \infty}\left(e^{i t H(V)} e^{-i t H_{0}-i X(p, t: V)} f, g\right) \\
& =\left(W^{(s)}(V) \mathscr{E}^{-1}\left[e^{-i X_{\infty 0}(\xi: V)} \widehat{f}(\xi)\right], g\right) .
\end{aligned}
$$

By Theorem 7.5 we have

$$
\begin{aligned}
(W(V) f, g)=\left(\Omega^{(s)}(V) \mathscr{F}^{-1}\left[e^{-i X_{\infty}(\xi: V)} \widehat{f}(\xi)\right], g\right) \\
\quad=\left(\mathscr{L}_{0} \mathscr{F}^{-1}\left[e^{-i X_{\infty}(\xi: V)} \widehat{f}(\xi)\right], \mathscr{F}^{(s)}(V) g\right) \widehat{\vartheta} \\
\quad=\int_{0}^{\infty}\left(\mathscr{F}_{0}(\lambda) \mathscr{F}^{-1}\left[e^{-i X_{\infty}(\xi: V)} \widehat{f}(\xi)\right], \mathscr{F}^{(s)}(\lambda: V) g\right)_{L_{2}(S n-1)} d \lambda .
\end{aligned}
$$

Recalling the definition of $\mathscr{F}_{0}(\lambda)$, we see that

$$
\begin{aligned}
\left(\mathscr{F}_{0}(\lambda) \mathscr{F}^{-1}\left[e^{-i X_{\infty}(\xi: V)} \hat{f}(\xi)\right]\right)(\omega) \\
=e^{-i X_{\infty}(\sqrt{\lambda} \omega: V)}\left(\mathscr{F}_{0}(\lambda) f\right)(\omega) .
\end{aligned}
$$

Therefore,

$$
\begin{aligned}
& (W(V) f, g)=\int_{0}^{\infty}\left(e^{-i X_{\infty}(\sqrt{\lambda} \cdot: V)} \mathscr{F}_{0}(\lambda) f, \mathscr{F}^{(s)}(\lambda: V) g\right)_{L_{2}\left(S^{n-1}\right)} d \lambda \\
& =\int_{0}^{\infty}\left(\mathscr{F}_{0}(\lambda) f, e^{i X_{\infty}(\sqrt{ } \bar{\lambda}:: V)} \mathscr{F}^{(s)}(\lambda: V) g\right)_{L_{2}\left(S^{n-1}\right)} d \lambda
\end{aligned}
$$

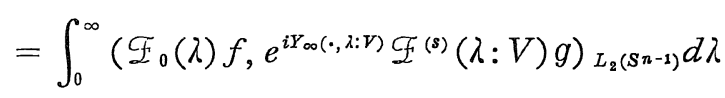

$$
\begin{aligned}
& \text { (by Lemma } 7.7 \text { ) } \\
& =\int_{0}^{\infty}\left(\mathscr{F}_{0}(\lambda) f, \mathscr{F}(\lambda: V) g\right)_{L_{2}\left(S^{n-1}\right)} d \lambda \\
& \text { (by Lemma } 7.8 \text { ) } \\
& =(\Omega(V) f, g) \text {. }
\end{aligned}
$$

§ 8. Proof of Theorem 1.1

Let $X(\xi, t: V)$ be as in Lemma 2.2. Let $\left\{V_{m}\right\}$ be a sequence in $\boldsymbol{V}=\boldsymbol{V}_{\delta}$ such that $V_{m}$ has compact support and $V_{m} \rightarrow V$ in $\mathbb{V}$. The existence of such a sequence $\left\{V_{m}\right\}$ is guaranteed by the fact that $\delta$ has been chosen less than $\delta_{0}$. Let $W(V), W\left(V_{m}\right), \Omega(V)$ and $\Omega\left(V_{m}\right)$ be the wave 
operators defined by using the above modifiers $X\left(\xi, t: V^{\top}\right)$ and $X\left(\xi, t: V_{m}\right)$. By Theorem 7.9 $W\left(V_{m}\right)=\Omega\left(V_{m}\right)$. On the other hand, since $W\left(V_{m}\right)$ $\rightarrow W(V)$ strongly by Theorem 2.6 , and since $\Omega\left(V_{m}\right) \rightarrow \Omega(V)$ strongly by Theorem 6.6, we can see that $W(V)=\Omega(V)$. The completeness of $W(V)$ follows from that of $\Omega(V)$ (Theorems 5.5 and 5.11). 圈

\section{Appendix, Construction of $X(\xi, t: \mathbb{V})$}

The purpose of this appendix is to give a proof of Lemma 2.2.

Hörmander [4] has obtained an exact solution of the equation

$$
\frac{\partial}{\partial t} X(\xi, t: V)=V\left(2 \xi t+\nabla_{\xi} X(\xi, t: V)\right)
$$

with a certain asymptotic condition at $\infty$. His solution $X(\xi, t: V)$ can be used as a time-dependent modifier, i.e. the properties enumerated in Lemma 2.2 are seen to be satisfied. We shall, however, state here another method of constructing a time-dependent modifier.

A successive approximation scheme for (9.1) is:

(9.2) $X^{(0)}(\xi, t: V)=0$,

$$
\begin{aligned}
& X^{(j)}(\xi, t: V)=\int_{0}^{t} V\left(2 \xi s+\nabla_{\xi} X^{(j-1)}(\xi, s: V)\right) d s+\phi_{j}(\xi: V), \\
& j=1,2, \cdots \\
& \phi_{j}(\xi: V)= \begin{cases}0 & \text { if } j \delta<1, \\
\phi_{j-1}(\xi: V)-\int_{0}^{\infty} A_{j-1}(\xi, s: V) d s \quad \text { if } j \delta>1,\end{cases} \\
& A_{j}(\xi, t: V)=V\left(2 \xi t+\nabla_{\xi} X^{(j)}(\xi, t: V)\right) \\
& \quad-V\left(2 \xi t+\nabla_{\xi} X^{(j-1)}(\xi, t: V)\right) .
\end{aligned}
$$

here we have used the fact that $1 / \delta$ is not an integer, which follows from our having chosen $\delta$ irrational.

Proposition A. $1 . X^{(j)}(\xi, t: V)$ is a real $C^{\infty}$-function of $\xi \neq 0$ and $t>0$ having the following properties: For an arbitrary compact set $K \subset \mathbb{R}^{n}-\{0\}$, we have for all $\xi \in K$ and $t>0$ 
(1) $\left|D_{\xi}^{a} D_{t}^{m} X^{(j)}(\xi, t: V)\right| \leq C(1+t)^{1-n-\grave{o}},|\alpha| \geq 0, m \geq 0$,

(2) $\left|D_{\xi}^{\alpha}\left(X^{(j)}(\xi, t: V)-X^{(j-1)}(\xi, t: V)\right)\right| \leq C(1+t)^{1-j o},|\alpha| \geq 0$,

(3) $\left|D_{\xi}^{\alpha} A_{j}(\xi, t: V)\right| \leq C(1+t)^{-(j+1) \dot{o}},|\alpha| \geq 0$,

(4) $\left|D_{\xi}^{\alpha} \phi_{j}(\xi: V)\right| \leq C$,

where the constanl $C$ does not depend on $V$ in a bounded set of $V$, but may depend on $j, \alpha, m$.

(5) If $V_{n} \rightarrow V$ in $V$, we have for all $\alpha$ and $m D_{\xi}^{\alpha} D_{t}^{m} X^{(j)}\left(\xi, t: V_{n}\right)$ $\rightarrow D_{\xi}^{\alpha} D_{t}^{m} X^{(j)}(\xi, t: V)$ pointwise for $\xi \neq 0$ and $t>0$ as $n \rightarrow \infty$.

Proof (by induction on $j$ ). First let us prove (1). (1) is true for $j=0$, since $X^{(0)}(\xi, t: V)=0$ by definition. Assume (1) when $j=k$. We have by definition

$$
\begin{aligned}
D_{\xi}^{\alpha} X^{(k+1)}(\xi, t: V)=\int_{0}^{t} D_{\xi}^{\alpha} & {\left[V\left(2 \xi s+\nabla_{\xi} X^{(k)}(\xi, s: V)\right)\right] d s } \\
& +D_{\xi}^{a} \phi_{k+1}(\xi: V) .
\end{aligned}
$$

$V \in \mathbb{V}$ and (1) when $j=k$ give

$$
\left|D_{\xi}^{\alpha}\left[V\left(2 \xi s+\nabla_{\xi} X^{(k)}(\xi, s: V)\right)\right]\right| \leq C(1+s)^{-\grave{ }} .
$$

On the other hand, by (3) and (4) when $j=k$ we get $\left|D_{\xi}^{\alpha} \phi_{k+1}(\xi: V)\right|$ $\leq C$, which proves (4). Hence we have

$$
\begin{aligned}
\left|D_{\xi}^{\alpha} X^{(k-1)}(\xi, t: V)\right| & \leq C \int_{0}^{t}(1+s)^{-\imath} d s+C \\
& \leq C(1+t)^{1-\hat{\jmath}}
\end{aligned}
$$

which proves (1) when $m=0$ with $j=k+1$. (The dependence of $C$ on $V$ and $\xi$ can be checked easily.) The case $m \geq 1$ can be treated in a similar way by differentiating (9.3) with respect to $t$.

Next we show that (1) and (2) imply (3). In fact, putting

$$
\begin{array}{r}
B_{j}(\xi, t: \theta: V)=\left(\nabla_{\xi} V\right)\left(2 \xi t+\theta \nabla_{\xi} X^{(j)}\left(\frac{\pi}{5}, t: V\right)\right. \\
\left.+(1-\theta) \nabla_{\xi} X^{(j-1)}(\xi, t: V)\right), \\
C_{j}(\xi, t: V)=\nabla_{\xi} X^{(j)}(\xi, t: V)-\nabla_{\xi} X^{(j-1)}(\xi, t: V),
\end{array}
$$

we have 


$$
\begin{aligned}
& A_{j}(\xi, t: V) \\
& =\int_{0}^{1} \frac{\partial}{\partial \theta} V\left(2 \xi t+\theta \nabla_{\xi} X^{(j)}(\xi, t: V)+(1-\theta) \nabla_{\xi} X^{(j-1)}(\xi, t: V)\right) d \theta \\
& =\int_{0}^{1} B_{j}(\xi, t: \theta: V) C_{j}(\xi, t: V) d \theta, \\
& D_{\xi}^{\alpha} A_{j}(\xi, t: V) \\
& =\int_{0}^{1} \sum_{\beta \leq \alpha}\left(\begin{array}{l}
\alpha \\
\beta
\end{array}\right) D_{\xi}^{\beta} B_{j}(\xi, t: \theta: V) D_{\xi}^{\alpha-\beta} C_{j}(\xi, t: V) d \theta \\
& \text { (Leibniz' formula). }
\end{aligned}
$$

since (1) and (2) yield for $\xi \in K, t>0$

$$
\begin{aligned}
& \left|D_{\xi}^{\alpha} B_{j}(\xi, t: \theta: \nabla)\right| \leq C(1+t)^{-1-\delta}, \\
& \left|D_{\bar{\xi}}^{a-\beta} C_{j}(\xi, t: V)\right| \leq C(1+t)^{1-j \hat{o}},
\end{aligned}
$$

we have $\left|D_{\tilde{\xi}}^{\alpha} A_{j}(\bar{\xi}, t: V)\right| \leq C(1+t)^{-(j+1) \dot{\delta}}$, which proves (3). Thus, in order to prove (2) and (3), we have only to prove (2) with $j=k+1$.

Let $N$ be the integer such that $N \delta<1$ and $(N+1) \delta>1$. We have

$$
\begin{aligned}
& D_{\xi}^{\alpha}\left(X^{(k+1)}(\xi, t: V)-X^{(k)}(\xi, t: V)\right) \\
&= \begin{cases}D_{\xi}^{\alpha} \int_{0}^{\iota} A_{k}(\xi, s: V) d s & (k<N), \\
-D_{\xi}^{\alpha} \int_{\imath}^{\infty} A_{k}(\xi, s: V) d s & (k \geq N) .\end{cases}
\end{aligned}
$$

Using (3) with $j=k$ we see that

$$
\left|D_{\xi}^{\alpha}\left(X^{(k+1)}(\xi, t: V)-X^{(i)}(\xi, t: V)\right)\right| \leq C(1+t)^{1-(k+1) j},
$$

which proves (2) for $j=k+1$.

The proof of (5) is easy and straightforward. 圈

Proof of Lemma 2.2. We choose the smallest positive integer $j$ such that $(j+1) \delta \geq 2$ and set $X(\hat{\xi}, t: V)=X^{(j)}\left(\xi, t: V^{\gamma}\right)$. By definition

$$
\frac{\partial}{\partial t} X(\xi, t: V)-V\left(2 \xi t+\nabla_{\xi} X(\xi, t: V)\right)=-A_{j}(\xi, t: V) .
$$

Thus all the assertions follow from Proposition A. 1. 㳡 


\section{References}

[1] Alsholm, P., Wave operators for long-range scattering, J. Math. Anal. Appl. 59 (1977) $550-572$.

[2] Alsholm, P., and Kato. T., Scattering with long-range potentials, Proc. Sympo. Pure Math., American Mathematical Society, Providence, R. I., 23, (1973) 393-399.

[3] Buslaev, V. S., and Matveev, V. B., Wave operators for the Schrödinger equation with a slowly decreasing potential, Theor. Math. Phys. 2 (1970), 266-274 (English translation from Russian).

[4] Hörmander, L., The existence of wave operators in scattering theory, Math. Z. 146 (1976), 69-91.

[5] Ikebe, T., Spectral representations for the Schrödinger operators with long-range Potentials, J. Funtional. Anal. 20 (1975), 158-177.

[6] Ikebe, T. and Saitō, Y., Limiting absorption method and absolute continuity for the Schrödinger operator, J. Math. Kyoto Univ. 12 (1972), 513-542.

[7] Isozaki, H., On the long-range stationary wave operator, Publ. RIMS, Kyoto Univ. 13 (1977), 589-626.

[8] Kato, T. and Kuroda, S. T., Abstract theory of scattring, Rocky Mt. J. Math. 1 (1971), 127-171.

[9] Kitada, H., On the completeness of modified wave operators, Proc. Japan Acad. 52 (1976), 409-412.

[10] , Scattering theory for Schrödinger operators with long-range potentials I, II, J. Math. Soc. Japan 29 (1977), 665-691 and 30 (1978), 603-632.

[11] Saitō, Y., Eigenfunction for the Schrödinger operators with long-range potentials $Q(y)=O\left(|y|^{-\varepsilon}\right) \quad(\varepsilon>0)$, Osaka J. Math. 14 (1977), 11-35.

[12] Titchmarsh, E. C., Eigenfunction expansions associated with second-order differential equations, part II, Oxford, Clarendon, 1958.

[13] Watson, G. N., Theory of Bessel functions, Cambridge, Cambridge Univ. Press., 1922.

[14] Yosida, K., Functional Analysis, Springer, Berlin-Heidelberg-New York, 1968. 